\title{
Dalle bacchette da calcolo cinesi al metodo \\ Fangcheng: un percorso di trasposizione culturale nella scuola secondaria di primo grado
}

\author{
From Chinese counting rods to Fangcheng method: \\ an experience of cultural transposition in lower \\ secondary school
}

\author{
Raffaele Casi $^{\star}$ e Chiara Pizzarelli ${ }^{\circ}$ \\ ^Istituto Comprensivo Andezeno - Andezeno (TO), Italia \\ ${ }^{\circ}$ Istituto Comprensivo Torino II E. Morelli - Torino, Italia
}

Sunto / Abbiamo proposto ad una classe prima di scuola secondaria di primo grado un percorso di scoperta di problemi della tradizione matematica cinese, riconducibili a sistemi di equazioni e risolubili mediante le antiche bacchette da calcolo e il metodo del Fangcheng. La metodologia proposta è la didattica laboratoriale, con l'uso di artefatti, quali bacchette e bilance, e di supporti audio-video. Sotto la lente teorica dell'analisi semiotica degli artefatti (Rabardel, 1999; Bartolini Bussi, Mariotti \& Ferri, 2005) e della trasposizione culturale (Bartolini Bussi, Sun \& Ramploud, 2013; Mellone \& Ramploud, 2015) si intendono mostrare le potenzialità di un uso formativo di antichi strumenti e tecniche di calcolo, provenienti da un contesto culturale, storico e geografico differente.

L'obiettivo è ripensare e aumentare la consapevolezza degli studenti sugli algoritmi di addizione e sottrazione e avviare all'utilizzo di metodi pre-algebrici fondati sul concetto di uguaglianza e sui principi di equivalenza.

Parole chiave: trasposizione culturale; storia della matematica cinese; artefatti; Early Algebra; Fangcheng.
Abstract / We proposed, to a class of sixth-graders, a path of discovery of Chinese mathematical tradition and its typical problems, akin to system of equations, to be solved with counting rods and the ancient method Fangcheng. The proposed methodology is laboratory didactics, using artifacts such as rods and scales as well as audio-video supports. According to the theory of the semiotic analysis of artifacts (Rabardel, 1999; Bartolini Bussi, Mariotti \& Ferri, 2005) and the theory of cultural transposition (Bartolini Bussi, Sun \& Ramploud, 2013; Mellone \& Ramploud, 2015), we intend to demonstrate the potential of a formative use of ancient instruments and counting techniques, ailing from a different cultural, historical and geographical context. The aim is to rethink first, and then boost, students' awareness about addition and subtraction algorithms while also directing them toward the use of pre-algebraic methods based upon the concept and principles of equivalence.

Keywords: cultural transposition; history of Chinese mathematics; artifacts; Early Algebra; Fangcheng.

\section{1}

Introduzione

Il nostro lavoro intende riprendere e fornire nuovi spunti ad alcune ricerche incentrate sull'utilizzo del metodo cinese del Fangcheng per risolvere sistemi di equazioni. In particolare, abbiamo tratto ispirazione dal lavoro di Costa, Alves e Guerra (2015), che hanno proposto a uno studente portoghese di 10 anni di risolvere in autonomia un problema con tale metodo, usando le cifre indo-arabiche; e dalla ricerca di Bagni (2006; 2009), che ha svolto un'analisi semiotica dell'artefatto che le bacchette cinesi offrono e ha sperimentato con esse l'applicazione della procedura del Fangcheng in 
una classe di scuola secondaria di primo grado'. II nostro intento è di avvicinarci a quest'ultima ricerca, ampliando la prospettiva alla metafora concettuale della bilancia e analizzando la sperimentazione secondo la lente della trasposizione culturale. La scelta dell'argomento sul quale lavorare è inusuale per una classe prima: le Indicazioni nazionali per il curricolo della scuola dell'infanzia e del primo ciclo d'istruzione (MIUR, 2012) non prevedono espressamente come obiettivo di apprendimento la risoluzione di problemi lineari mediante I'utilizzo di sistemi di equazioni di primo grado, che è invece previsto come obiettivo specifico nel primo biennio della scuola secondaria di secondo grado ${ }^{2}$. Tuttavia, I'attività che proponiamo agli studenti ha un duplice intento: da un lato quello di rivedere le operazioni di addizione e sottrazione di numeri interi - che sono state ampiamente studiate alla scuola primaria ${ }^{3}$ - con un'ottica più matura, maggiormente legata a un utilizzo di tali operazioni in campo algebrico; dall'altro quello di stimolare gli studenti a comprendere come alcuni strumenti matematici (tra cui equazioni e sistemi di equazioni) siano funzionali alla risoluzione di problemi.

È invece cruciale, secondo le Indicazioni nazionali (MIUR 2012, p. 60), il ruolo del laboratorio, inteso come «momento in cui l'alunno è attivo, formula le proprie ipotesi [...], discute e argomenta le proprie scelte, negozia e costruisce significati, portando a conclusioni [...] e a nuove aperture la costruzione delle conoscenze personali e collettive». Intendiamo, dunque, utilizzare tale approccio, partendo da problemi concreti, tratti dalla storia della matematica cinese e del suo insegnamento, e facendo uso di artefatti. L'obiettivo finale è di radicare un senso per i classici metodi di risoluzione di sistemi di equazioni lineari, che talvolta sono appresi meccanicamente in livelli scolari superiori. Il percorso qui proposto consiste in primis nella scoperta da parte degli studenti di simboli numerici usati nell'antica Cina, tramite l'analisi di una pagina di un antico libro di testo di matematica cinese, il "Prezioso specchio dei quattro elementi" (1303), in cui è rappresentato il cosiddetto "triangolo di Tartaglia-Pascal". Una volta introdotto il sistema di numerazione e gli algoritmi dell'addizione e della sottrazione, osservati tramite video e poi replicati fisicamente con le bacchette e tavole da calcolo, ci siamo concentrati sul capitolo VIII del trattato "I Nove Capitoli sui procedimenti matematici" (I sec. a.C. - I sec. d.C.). Esso, infatti, offre un'ampia varietà di problemi, riconducibili a sistemi di equazioni di primo grado a due o più incognite, risolubili tramite il metodo cinese del Fangcheng. Per comprendere tale antico metodo, sono state previste attività che, partendo dall'esempio fisico della doppia bilancia, hanno gradualmente portato all'astrazione e pre-formalizzazione algebrica del metodo.

\section{Contesto storico: la matematica nell'antica Cina}

Prima di affrontare le fasi del percorso, riteniamo utile fornire alcune indicazioni sul sistema di numerazione dell'antica Cina, che faceva uso delle bacchette e tavole da calcolo, e sul metodo del Fangcheng.

1. La scuola secondaria di primo grado italiana corrisponde ai primi tre anni di scuola media nel Canton Ticino. 2. Il primo biennio della scuola secondaria di secondo grado italiana corrisponde alla quarta media e al primo anno di scuola media superiore o di scuola professionale nel Canton Ticino.

3. La scuola primaria italiana corrisponde alla scuola elementare nel Canton Ticino. 
Dalle bacchette da calcolo cinesi al metodo Fangcheng: un percorso di trasposizione culturale nella scuola secondaria di primo grado / Raffaele Casi e Chiara Pizzarelli

\subsection{Numeri e scrittura nell'antica Cina}

Dalla dinastia Han (206 a.C. - 220 d.C.) fino alla dinastia Yuan (XIII secolo) in Cina le principali tecniche di calcolo si basavano sull'uso di bacchette da calcolo: bastoncini di bambù o avorio di circa 2,5 mm di diametro e $15 \mathrm{~cm}$ di lunghezza, facilmente manovrabili per eseguire operazioni aritmetiche. Le bacchette, disposte su tavole, talvolta quadrettate, hanno rappresentato per secoli il principale strumento di calcolo pratico. II loro uso cessò gradualmente nella dinastia dei Ming (1368-1644), quando furono soppiantate dal suanpan, I'abaco cinese.

La scrittura dei numerali, ossia i segni che rappresentano quantità - risalenti al I millennio a.C. - era piuttosto intuitiva, facendo probabilmente riferimento alle dita della mano: i numeri 1, 2, 3, 4 e 5 si rappresentavano affiancando rispettivamente $1,2,3$, 4 e 5 bacchette verticali; i numeri da 6 a 9 si rappresentavano con un'asta in orizzontale a indicare cinque unità e sotto di essa tante aste verticali quante occorre aggiungere per completare il numero. Per la rappresentazione delle decine si utilizzavano simboli analoghi, ma con le bacchette disposte in orizzontale (Figura 1).

\section{Figura 1 \\ Rappresentazione dei numeri nelle disposizioni Tsung (sopra) e Heng} (sotto) nell'antica Cina.

\begin{tabular}{|c|c|c|c|c|c|c|c|c|}
\hline | & $\|$ & ||| & ||l| & ||l|| & $T$ & $\pi$ & TाI & TाI \\
\hline 1 & 2 & 3 & 4 & 5 & 6 & 7 & 8 & 9 \\
\hline - & $=$ & $\equiv$ & $\equiv$ & $\equiv$ & $\perp$ & $\perp$ & $\perp$ & $\perp$ \\
\hline 10 & 20 & 30 & 40 & 50 & 60 & 70 & 80 & 90 \\
\hline
\end{tabular}

Per gli ordini di grandezza successivi si continuava ad alternare la disposizione verticale (Tsung), valida quindi per unità, centinaia, decine di migliaia ecc., con quella orizzontale (Heng), per decine, migliaia, centinaia di migliaia ecc. II sistema era decimale posizionale: le bacchette erano affiancate mantenendo nella colonna di destra quelle rappresentanti le unità, seguite a sinistra dai successivi ordini numerici (Figura 2).

Almeno fino all'VIII secolo d.C. non sono noti simboli per lo zero, che si rappresentava sulla tavola lasciando la cella vuota o - nel testo scritto - uno spazio; successivamente fu introdotto un piccolo cerchio, derivato dalla diffusione del sistema posizionale indiano.

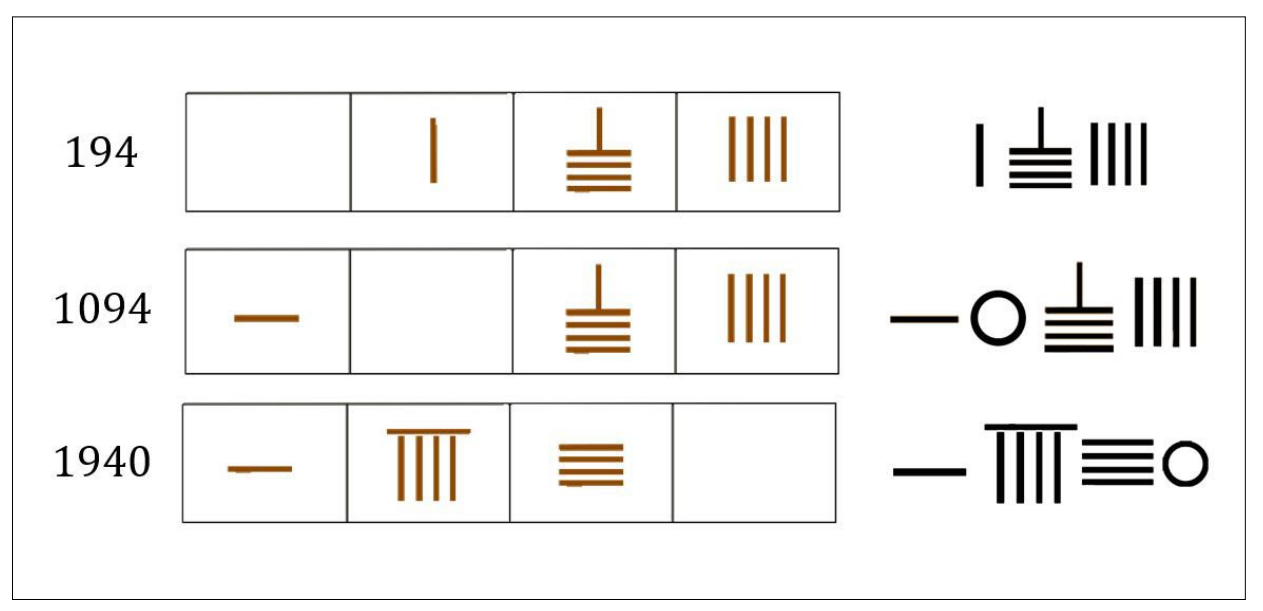


Figura 3

Algoritmo cinese dell'addizione. Nell'esempio:

$183+231=414$
Si tratta di un sistema di scrittura operatorio, ossia la forma di rappresentazione del numero conserva l'effettiva somma dei simboli che lo compongono; e avente un carattere di immanenza più esplicito rispetto alle scritture moderne (Bagni, 2009), ovvero il simbolo fornisce un'immagine concreta del numero che rappresenta (Martzloff, 2006). I numerali (così come gli ideogrammi per le parole) rappresentano concretamente il valore (e il significato) del numero (e della parola). II numero 3 è indicato con tre linee verticali (così come, ad esempio, la parola cavallo è una stilizzazione del disegno di un cavallo). Diversamente accade per la notazione occidentale che si è strutturata nei secoli seguendo processi di astrazione (il simbolo 3 non ha nessun legame con il valore numerico che rappresenta).

Eseguire un'addizione fra due numeri consiste nel rappresentare gli addendi su due righe della tavola da calcolo, separate da una riga vuota, nella quale vengono successivamente fatte slittare le bacchette degli addendi senza cambiare colonna. Dopo aver opportunamente aggiustato i bastoncini con la corretta rappresentazione del numero ed eventuali riporti, si legge la somma (Figura 3).

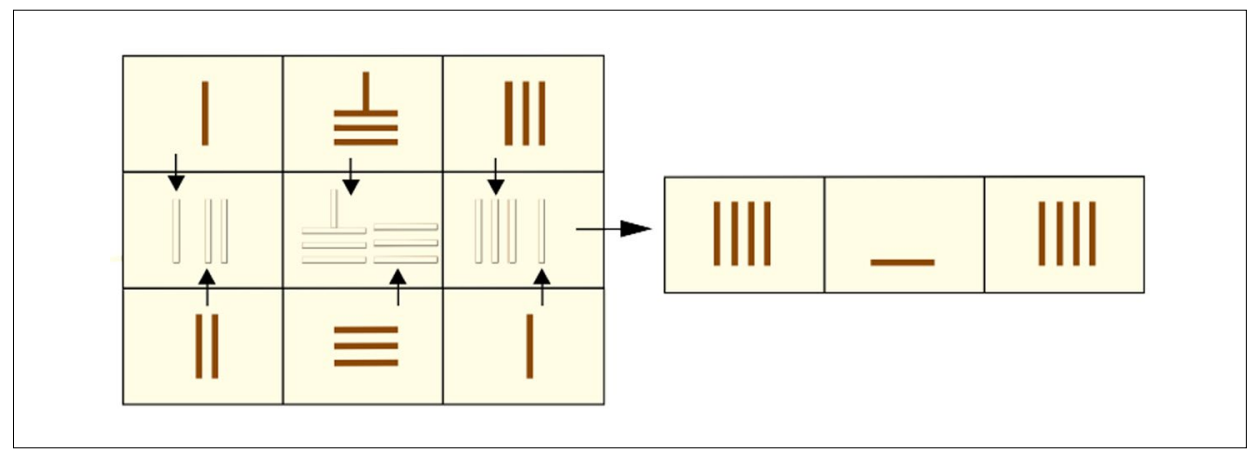

Per quanto riguarda invece l'algoritmo della sottrazione, si procedeva nel seguente modo: si disponeva il minuendo nella riga in alto della tavola e successivamente si rappresentava il sottraendo nella riga sottostante, attingendo dalle bacchette del minuendo, scegliendo dalla cella delle unità per rappresentare le unità, dalle decine per le decine e così via. In questo modo nella prima riga si poteva leggere il risultato della sottrazione. Nel caso in cui non fosse presente una quantità sufficiente di bacchette nell'ordine di grandezza corrispondente, si rendeva necessario un prestito, ossia una trasformazione dall'ordine di grandezza più elevato, eliminando ad esempio una decina per far comparire dieci unità (Figura 4).

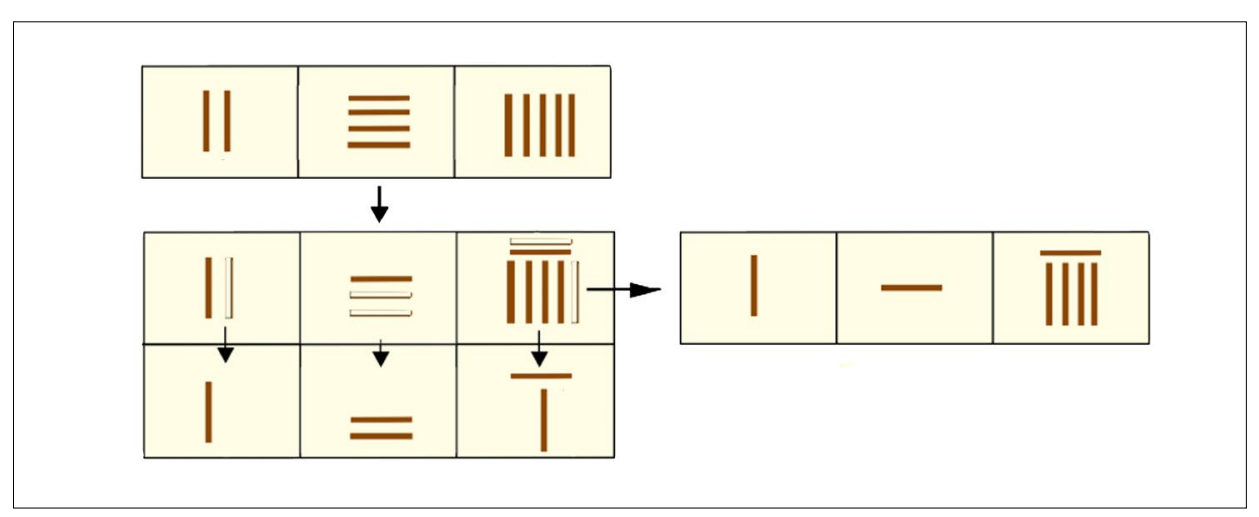


Figura 5

In questa immagine si può osservare la diffe-

renza tra l'algoritmo

dell'addizione odierno e quello dell'antica Cina.
Dal confronto tra gli algoritmi di addizione e sottrazione con le bacchette e quelli attuali, possiamo osservare come le procedure di riporto e prestito con le bacchette esplicitino in modo più evidente le quantità da spostare negli ordini di grandezza superiore e inferiore. Nello specifico, nel caso dell'addizione la numerosità delle bacchette permette di vedere a colpo d'occhio se una certa quantità è superiore a 10 , e quindi è da riportare. Ad esempio, volendo sommare 26 e 17 con l'algoritmo odierno dell'addizione procediamo così: $6+7=13$, scrivo 3 riporto 1 (Figura 5).

In tale procedura non è evidente che quell'uno riportato sia in realtà un 10. Le bacchette cinesi invece ci mostrano la presenza di una grandezza superiore a 9 e ci obbligano a riportare (fisicamente) due volte cinque - cioè proprio un dieci - nell'ordine di grandezza superiore.

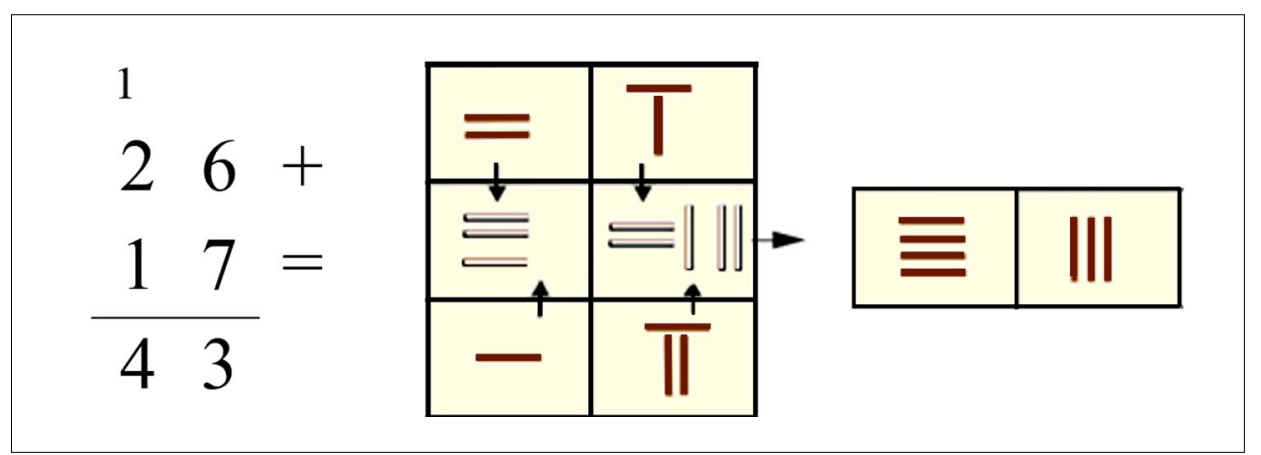

Un discorso analogo vale per la sottrazione, dove si nota come la tecnica cinese si riveli particolarmente efficiente in caso di differenze senza prestito (ad esempio nella sottrazione 27-20 occorre togliere le due bacchette orizzontali delle due decine), mentre negli altri casi comporta un ragionamento sui "valori che formano il prestito". Ad esempio, per effettuare la sottrazione 32-17 con l'algoritmo odierno ragioneremmo così: «poiché 2-7 non si può fare, mi faccio prestare un 1 dal 3, che diventa 2, e il 2 diventa 12; quindi 12-7=5, 2-1=1» (Figura 6). Anche in questo caso non è evidente che I'1 sia un 10 , che va sommato al 2 . Con le bacchette da calcolo, invece, è necessario spostare una decina dalla seconda colonna, e trasformarla in $5+5$ nella prima. Quindi si procede prendendo le bacchette dalla prima riga e spostandole nella seconda. Infine, si effettua un aggiustamento sul cinque, che va espresso correttamente con cinque bacchette verticali.

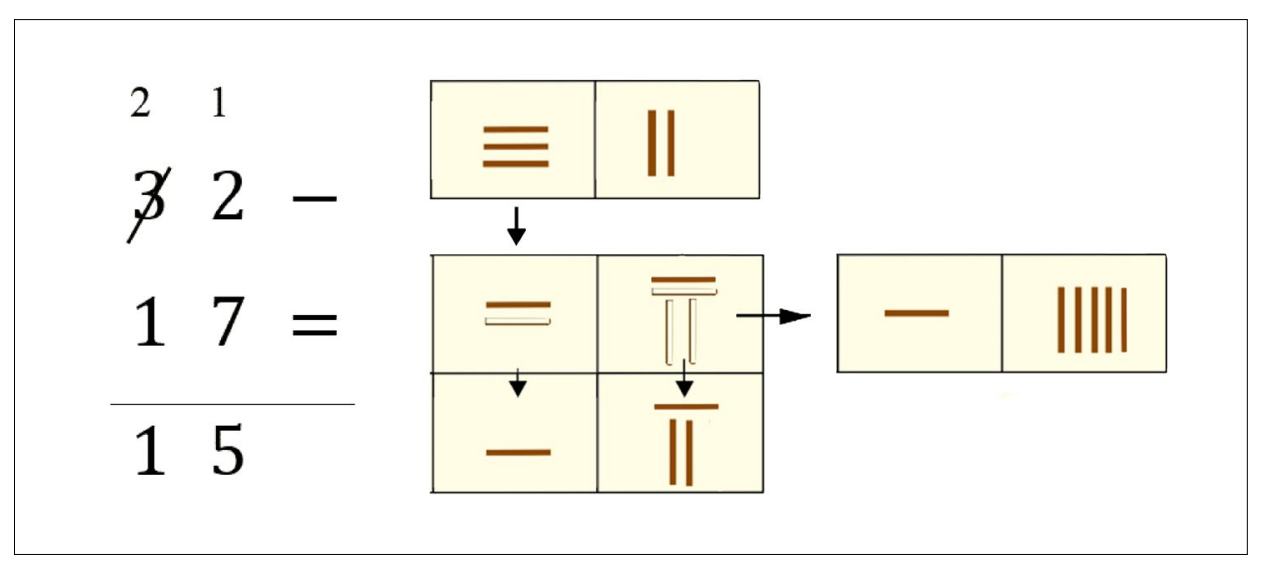


L'algoritmo dell'addizione, insieme ad altre tecniche aritmetiche, geometriche ed algebriche, è descritto nel più antico testo matematico cinese ad oggi noto "I Nove Capitoli sui procedimenti matematici" (Jiuzhang suanshu, I sec. a.C. - I sec. d.C.), anonimo. Oggetto di numerosi successivi commentari, il manuale ebbe un'influenza sulla matematica cinese paragonabile a quella degli Elementi di Euclide in Occidente. II testo contiene 246 problemi pratici, suddivisi a seconda degli algoritmi risolutivi (Chemla \& Shuchun, 2004).

Un altro importante trattato cinese è il "Prezioso specchio dei quattro elementi" (Siyuan Yujian o Ssu Yuan Yu Chien, 1303), ${ }^{4}$ di Zhu Shijie, che rappresenta il «punto più alto raggiunto dall'algebra cinese» (D'Amore \& Sbaragli, 2018, p. 138). Per la nostra sperimentazione ci siamo valsi di un'immagine selezionata da tale trattato, che rappresenta quello che noi chiamiamo il triangolo di Tartaglia-Pascal. Senza far menzione della paternità, Zhu Shijie descrive la sua "Tavola dell'antico metodo per elevare all'ottava potenza". La procedura per la costruzione della tavola dei coefficienti binomiali era stata descritta in precedenza già da Jia Xian (1050 circa), ma è probabile che l'idea di fondo del triangolo fosse antecedente (Martzloff, 2006). Siamo dunque di fronte ad un concetto matematico cui noi siamo soliti attribuire la nascita all'interno della nostra cultura occidentale, ma che è stato sviluppato in maniera indipendente in un altro contesto, in un'altra area geografica e probabilmente anche in un'epoca precedente. Ciò rende il triangolo dei coefficienti binomiali cinese un importante strumento per ripensare a quel carattere di unicità della storia della matematica, cui i nostri studenti - e non solo - sono abituati.

\subsection{Il metodo del Fangcheng}

L'antica procedura per la risoluzione di sistemi di equazioni lineari, detta Fangcheng ${ }^{5}$ è descritta ne "I Nove Capitoli sui procedimenti matematici". Assimilabile all'odierno metodo di eliminazione gaussiana per sistemi di equazioni, essa si basa su un'impostazione matriciale, in cui ogni colonna contiene i coefficienti numerici di una stessa incognita e l'ultima colonna i termini noti.

II lettore potrà chiedersi: per quale motivo dover introdurre in una scuola secondaria di primo grado notazioni che appartengono a gradi scolastici ben superiori? Le ragioni sono da ritrovare nel carattere posizionale della storia dell'algebra cinese. Essa si concentrava su un'impostazione matriciale per via delle tavole da calcolo che i matematici, e non solo, utilizzavano anche per i più semplici algoritmi aritmetici; ma non si fondava sul concetto di determinante, sviluppatosi in Oriente solo nel 1683 (Bagni, 2006). Ciò rende I'artefatto particolarmente intuitivo e basato su un approccio aritmetico elementare. Inoltre, I'utilizzo della tavola da calcolo quadrettata per la risoluzione di sistemi di equazioni rende superfluo l'uso di un simbolismo algebrico per le incognite: determinate posizioni nella tavola corrispondono, infatti, a particolari tipi di grandezze. Nella nostra attività abbiamo considerato il seguente problema, ripreso da una sperimentazione in cui Bagni utilizza la procedura Fangcheng di "eliminazione per moltiplicazione reciproca".

4. I quattro elementi (cielo, terra, uomo e materia) sono le quattro incognite di un'equazione.

5. La parola Fangcheng, derivante da fang, "mettere fianco a fianco", e cheng, "misura, norma delle cose", può essere tradotta con "cercare le norme delle cose assemblandole fianco a fianco". L'espressione rimanda all'insieme di relazioni quantitative tra cose che sono affiancate, e alla procedura che porta a valutare le norme delle misure di ciascuna cosa. Per approfondimenti cfr. Di Paola e Spagnolo (2009, pp. 113-115). 
Figura 7

Rappresentazione del

sistema di equazioni line-

ari che risolve il problema,

mediante notazione odier-

na con sistemi e matrice

dei coefficienti (a sinistra)

e mediante la tavola da

calcolo dell'antica Cina (a

destra), leggermente modi-

ficata per i nostri scopi

didattici (etichette in alto e

separazione di coefficienti

e termini noti).
Cinque casse uguali di grano aggiunte a tre sacchi uguali di grano pesano $19 \mathrm{~kg}$.

Tre casse uguali di grano aggiunte a due sacchi uguali di grano pesano $12 \mathrm{~kg}$.

Quanti kg pesa una cassa di grano e un sacco di grano?

II testo è liberamente tratto da un problema del capitolo VIII de "I Nove Capitoli sui procedimenti matematici". Abbiamo scelto di riprendere tale esempio perché a livello operativo non comporta particolari difficoltà nel calcolo con le bacchette e agevola la comprensione della procedura. Tuttavia, abbiamo preferito adottare la cosiddetta "nuova procedura Fangcheng", che si fonda su sottrazioni ripetute, perché, come si vedrà nelle sezioni successive, risulta efficace nell'utilizzo della metafora concettuale delle bilance a due piatti.

Il problema in notazione moderna si riconduce ad un sistema di equazioni. Per applicare il Fangcheng si posizionano i coefficienti ed i termini noti in due righe della tabella da calcolo. In realtà, nei trattati cinesi si posizionano lungo colonne, ma scegliamo di modificare l'orientamento per rendere più semplice il collegamento con i sistemi di equazioni che gli studenti studieranno in anni successivi (Figura 7).

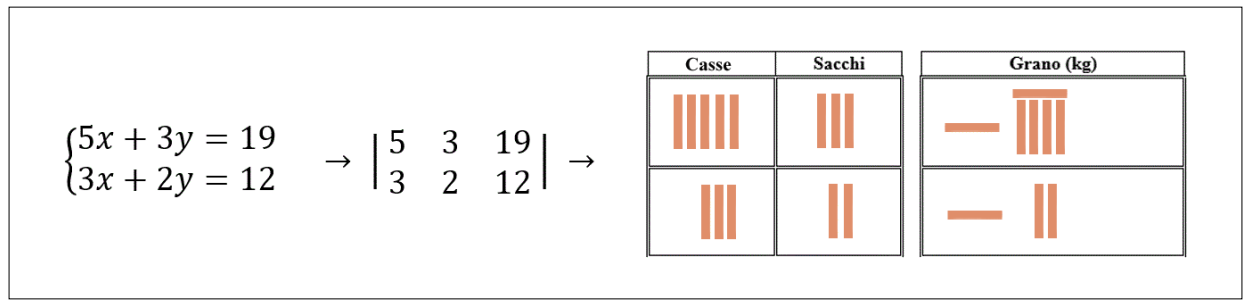

II metodo del Fangcheng consiste nel modificare le righe della tabella in modo tale che la matrice dei coefficienti sia diagonale, ossia abbia solamente i valori della diagonale principale non nulli. In questo modo, da relazioni quantitative tra due incognite, se ne ottiene il valore numerico. Per modificare le righe, il Fangcheng prevede di adottare due regole:

1. si possono moltiplicare o dividere per uno stesso numero non nullo tutti i termini di una riga;

2. si possono sostituire i termini di una riga con quelli ottenuti dall'addizione o sottrazione dei corrispondenti termini di altre righe.

Nella sperimentazione abbiamo scelto di avviare gli studenti al metodo con esercizi che richiedessero solamente l'applicazione della regola 2. Per agevolare il lettore, illustriamo qui di seguito i principali passaggi della risoluzione del sistema mediante la "nuova procedura Fangcheng" (Figura 8). 
Figura 8

Risoluzione del sistema di equazioni lineari con il metodo di eliminazione per sottrazioni ripetute (nuova procedura del

Fangcheng): nel passaggio 2 si sottraggono i termini della seconda riga dalla prima e si inseriscono le differenze nella prima riga; nel passaggio 3 si sottraggono i termini della prima riga dalla seconda e si inseriscono le differenze nella seconda riga. Si procede analogamente fino ad annullare il coefficiente di un'incognita per ciascuna riga.
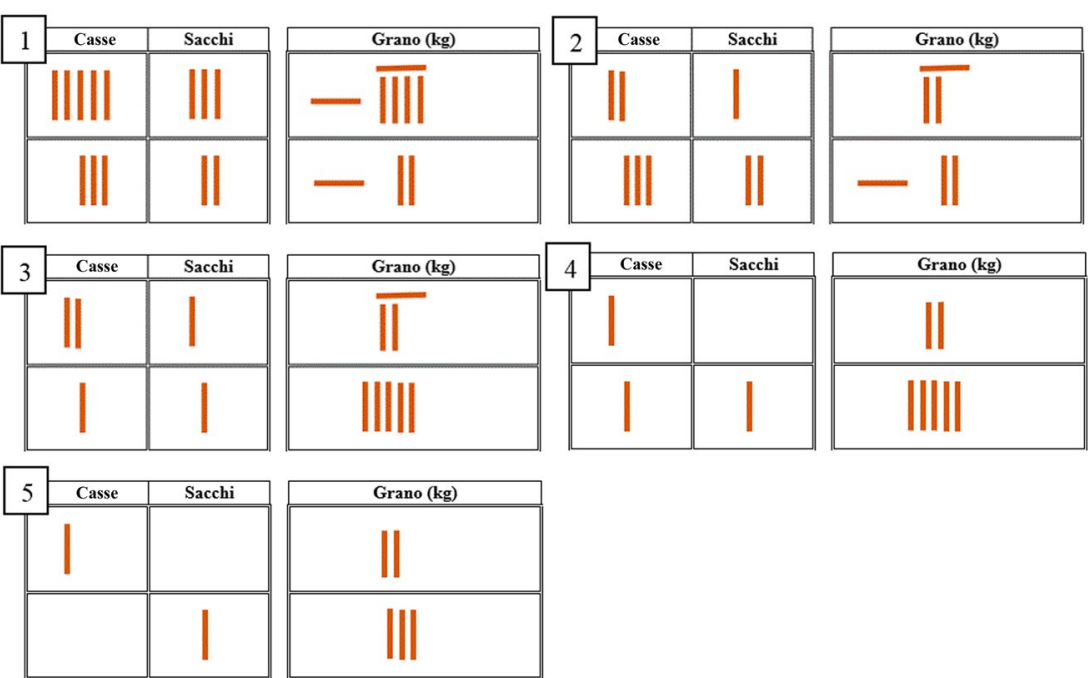

\section{3 ameromence}

In questa sezione illustriamo le lenti teoriche che hanno guidato la progettazione metodologica del nostro percorso didattico.

\subsection{La trasposizione culturale e la storia delle matematiche}

In una scuola multietnica e alla continua ricerca di strumenti inclusivi, I'approccio interculturale si può rivelare assai produttivo didatticamente. Per la progettazione di tale attività ci siamo, pertanto, valsi del quadro teorico della trasposizione culturale (Bartolini Bussi, Sun \& Ramploud, 2013; Mellone \& Ramploud, 2015). Il costrutto definisce una condizione di decentramento dalla pratica didattica del proprio contesto culturale, passando attraverso il contatto con pratiche didattiche di altri contesti culturali (Mellone, Ramploud, Di Paola \& Martignone, 2019). Riteniamo che la nostra sperimentazione possa inserirsi in tale interessante quadro teorico, in quanto la nostra idea è quella di ripensare alle intenzionalità educative di testi didattici della storia della matematica cinese, con l'obiettivo di cercare nuove chiavi interpretative per la pratica didattica nel nostro contesto culturale. In particolare, osserveremo come sarà possibile generare possibilità di ragionamento differenti dalle usuali quando affronteremo con gli studenti un particolare sistema di rappresentazione dei numerali in uso nell'antica Cina, gli algoritmi dell'addizione e della sottrazione, e il metodo del Fangcheng per risolvere problemi.

Notiamo che uno degli aspetti della matematica cinese che rende più agevole il dialogo traspositivo tra le didattiche delle matematiche delle due culture è - come anticipato nel par. 2.1 - il carattere operativo e immanente della notazione numerica delle bacchette da calcolo usata nell'antica Cina. Tale aspetto, come vedremo nella sperimentazione, può rendere più intuitiva l'applicazione di alcune procedure aritmetiche e algebriche, aiutando a comprenderne più in profondità il funzionamento. Dall'altro lato, l'esecuzione di procedure su una tavola da calcolo non permette di 
tenere memoria dei passaggi effettuati; ciò rende gli algoritmi odierni più agevoli dal punto di vista del controllo operatorio.

In linea dunque con il quadro teorico della trasposizione culturale, con tale percorso si intende promuovere non solamente un avvicinamento, ma una vera e propria interazione tra diverse culture, al fine di valorizzarne le differenze e potenzialità. Come afferma Bachtin (2000), infatti:

\begin{abstract}
«Una certa immedesimazione nella cultura altrui [...] è un momento necessario del processo della sua comprensione; ma se la comprensione si esaurisse in questo solo momento, essa sarebbe una semplice duplicazione e non porterebbe in sé nulla di nuovo e di arricchente. La comprensione creativa non rinuncia a sé, al proprio posto nel tempo, alla propria cultura e non dimentica nulla».
\end{abstract}

(Bachtin, 2000, p. 347)

Il nostro approccio teorico alla trasposizione culturale mira a ricercare nuove pratiche didattiche in un contesto culturale diverso dal nostro non solo geograficamente, ma anche storicamente. Per questo motivo non possiamo prescindere dal considerare gli studi, ad oggi ben noti, sulle potenzialità dell'introduzione della Storia delle matematiche nell'insegnamento (Furinghetti \& Radford, 2002; Radford, Boero \& Vasco, 2000; Radford, 2003). L'accento posto sul duplice aspetto della storia e della geografia porta a riflettere sul fatto che non esista un'unica storia della matematica, ossia quella occidentale, come ben sottolinea Bagni (2006).

\title{
3.2 Artefatti come strumenti di mediazione semiotica
}

L'applicazione delle bacchette da calcolo richiede un'ulteriore precisazione sul quadro teorico di cui ci siamo avvalsi, rientrando nell'ampia ricerca sulle funzioni di mediazione semiotica degli artefatti. Come afferma Vygotskij,

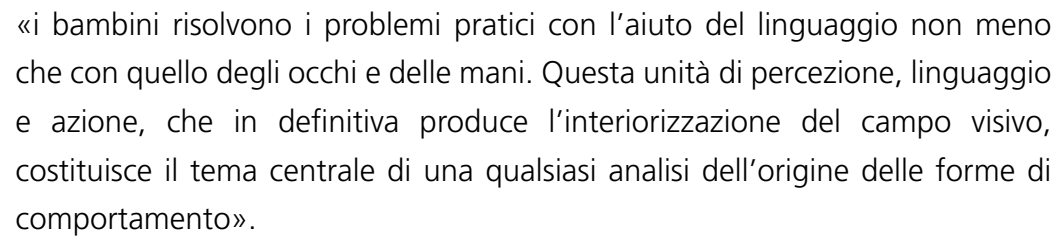

(Vygotskij, 1987, p. 26)

Nella sperimentazione oggetto di studio, facendo riferimento all'approccio strumentale di Rabardel (1999) e al quadro teorico proposto da Bartolini Bussi, Mariotti e Ferri (2005), le bacchette da calcolo, la pagina di un testo antico e le bilance si configurano come artefatti primari da manipolare. Le modalità per variare le tavole da calcolo e i video su tablet possono invece essere interpretati come artefatti secondari. La rappresentazione algebrica dei sistemi di equazioni è invece un artefatto terziario. Tali artefatti presentano un potenziale semiotico: il loro utilizzo può infatti produrre segni specifici e contribuire alla costruzione di significati matematici; diventano cioè strumenti di mediazione semiotica (Bartolini Bussi \& Mariotti, 2008). In particolare, nella nostra sperimentazione l'esplicita iconicità dei numerali cinesi e la presenza concreta delle bacchette ci consentono di evidenziare l'importanza dell'esperienza sensomotoria nella formazione di idee matematiche (Lakoff \& Núñez, 2000; Arzarello \& Robutti, 2009). Relativamente all'obiettivo didattico dell'acquisizione di maggior consapevolezza del 
prestito e del riporto negli algoritmi dell'addizione e della sottrazione, diventa cruciale instaurare una relazione tra gli artefatti (le bacchette, il testo antico e i video) e il compito (scoprire il funzionamento del sistema di numerazione cinese), espressa tramite segni (perlopiù parole, disegni e gesti deittici). Attraverso la discussione tra gruppi e della classe si intende far evolvere il significato dei segni, fino a ricondursi ai segni matematici già conosciuti e ricontestualizzati nella cultura di appartenenza, segni che in questo modo possono essere rielaborati, messi in discussione e, infine, compresi a livello concettuale.

In maniera analoga l'obiettivo didattico di avviare a metodi pre-algebrici nella risoluzione di sistemi di equazioni si fonda sull'utilizzo combinato delle bilance a due piatti, tavola da calcolo e bacchette. Anche in questo caso risulta importante il ruolo dei segni situati (disegni e gesti deittici) per avvicinarsi ai segni matematici (formalizzazione odierna di un sistema di equazioni). II Fangcheng è la procedura che gli studenti devono oggettualizzare, al fine di scoprirne, tramite gli artefatti, il funzionamento (con i video) e il senso fisico (con le bilance a due piatti), in modo da renderla un "oggetto autonomo" da applicare ad altri problemi analoghi.

Nel ciclo didattico (Bartolini Bussi \& Mariotti, 2008) da noi seguito gli studenti sono stati coinvolti in diverse attività che richiedevano l'uso di artefatti. L'iniziale interpretazione di un diagramma con segni scritti, rappresentante il triangolo di Tartaglia-Pascal con numerazione cinese, è seguita dall'utilizzo dell'artefatto, ossia le bacchette, per prendere confidenza con la rappresentazione dei numeri. Agli studenti è chiesto di produrre ipotesi sulla possibile rappresentazione dei numeri nell'antica Cina. Alla fase dell'attività con l'artefatto, è seguita la produzione di un testo a gruppi. Termina il ciclo la discussione matematica (Bartolini Bussi, Boni \& Ferri, 1995), collettiva, orchestrata dall'insegnante, parte essenziale del processo di insegnamento-apprendimento. Il ciclo didattico è poi ripreso per le attività successive: la richiesta di osservare un video che mostra gli algoritmi dell'addizione e della sottrazione adottati nell'antica Cina è seguita da una produzione a gruppi del resoconto di quanto osservato. L'obiettivo di tale ciclo è quello di concentrarsi sui processi semiotici di produzione ed elaborazione di segni legati alle attività con gli artefatti. Anche la terza fase della sperimentazione - ossia la scoperta del Fangcheng attraverso il video con le bilance ha rispettato il ciclo didattico da noi impostato. Per tale fase abbiamo infatti richiesto di osservare il video e di interagire con esso, quindi di descrivere dettagliatamente tutti i processi logici della procedura, anche avvalendosi dell'utilizzo delle bacchette. Qui gli artefatti che entrano in gioco sono due: le bacchette da calcolo, come già nelle fasi precedenti, e le due bilance a piatti, il cui schema di utilizzo è in parte noto agli studenti e in parte viene presentato nel video messo a disposizione. La scelta di utilizzare la metafora concettuale della bilancia per questa fase della sperimentazione deriva dal lavoro di Giacomin e Navarra (2003) sull'Early algebra, che propongono I'utilizzo delle bilance per lavorare sul concetto di uguaglianza all'interno del quadro teorico previsto per il progetto ArAl (Malara \& Navarra, 2003). La nostra sperimentazione, inoltre, si vale dei risultati dello studio di Mihajlović e Milikić (2019), che hanno evidenziato il ruolo del Fangcheng nello sviluppo di competenze pre-algebriche. Infine, sottolineiamo che l'idea di collegare questa tipologia di problemi alle bilance è presente anche ne "I Nove Capitoli sulle procedure matematiche" in uno dei problemi dell'VIII capitolo. ${ }^{6}$

6. «Problema 8.9: Supponiamo che 5 passeri e 6 rondini si riuniscano su di una bilancia e che l'insieme dei passeri sia più pesante dell'insieme delle rondini. Se un passero ed una rondine cambiano il loro posto, I'ago della bilancia è in orizzontale. Se si mettono assieme passeri e rondini, il peso è di 1 Jin. Si chiede quanto pesano rispettivamente un passero ed una rondine» (Di Paola \& Spagnolo, 2009, pp. 150-152). 


\section{Metodologia}

La sperimentazione è stata proposta nella prima parte dell'anno scolastico 2019/2020 nella classe I B della scuola secondaria di primo grado "G. Berruto" di Baldissero Torinese, facente parte dell'ı. C. Andezeno.

La classe, formata da 20 allievi, ha lavorato in aula, con due insegnanti-ricercatori (di cui uno titolare della classe), in gruppi eterogenei di 3-4 alunni. Nella sperimentazione si è scelto di alternare le attività di gruppo a discussioni matematiche orchestrate dall'insegnante. Le sessioni di lavoro sono state 10, di circa 1-2 ore ciascuna, per un totale di 15 ore, nell'arco di 4 settimane; documentate tramite video, foto e raccolta dei materiali e protocolli prodotti.

Abbiamo scelto di utilizzare una modalità laboratoriale per raccordare la prima parte della programmazione didattica, dedicata all'approfondimento e al ripasso delle operazioni aritmetiche, a una seconda parte, centrata sull'utilizzo dei problemi.

\section{Descrizione dell'itinerario didattico}

Il percorso da noi proposto è suddiviso nelle seguenti fasi: dapprima l'approccio ai numerali dell'antica Cina mediante le bacchette, in seguito I'utilizzo delle bacchette per eseguire addizioni e sottrazioni, infine la risoluzione di problemi con la tecnica del Fangcheng, introdotta con I'utilizzo della metafora concettuale delle bilance. Al termine di questo itinerario è stata proposta un'attività di verifica degli apprendimenti e un questionario di valutazione del percorso svolto.

La Tabella 1 riassume il percorso didattico progettato e attuato, indicando gli obiettivi di ciascuna fase, le risorse e i tempi utilizzati. Per rendere più fruibile la lettura di questo articolo, abbiamo ritenuto di descrivere le attività senza riportare le schede di lavoro utilizzate, che sono disponibili in allegato.

\begin{tabular}{|l|l|l|l|}
\hline Attività & Obiettivo & Risorse & Durata \\
\hline $\begin{array}{l}\text { 1. I numerali nell'antica } \\
\text { Cina }\end{array}$ & $\begin{array}{l}\text { Scoprire un sistema di } \\
\text { rappresentazione dei } \\
\text { numeri nell'antica Cina. }\end{array}$ & $\begin{array}{l}\text { Allegato 1 con il triangolo dei } \\
\text { coefficienti binomiali tratto dal } \\
\text { "Prezioso Specchio dei Quattro } \\
\text { Elementi". }\end{array}$ & 3 ore \\
\hline $\begin{array}{l}\text { 2. Le operazioni con } \\
\text { bacchette e tavola } \\
\text { da calcolo }\end{array}$ & $\begin{array}{l}\text { Padroneggiare gli algorit- } \\
\text { mi di addizione e sottra- } \\
\text { zione con le bacchette ci- } \\
\text { nesi e la tavola da calcolo. }\end{array}$ & $\begin{array}{l}\text { Allegati 2 e 3. Bacchette e tavole } \\
\text { da calcolo. Video che mostra } \\
\text { I'applicazione degli algoritmi, } \\
\text { proiettato su LIM. }\end{array}$ & 4 ore \\
\hline $\begin{array}{l}\text { 3. La procedura del } \\
\text { Fangcheng }\end{array}$ & $\begin{array}{l}\text { Scoprire l'antico metodo } \\
\text { cinese per la soluzione } \\
\text { di sistemi di equazioni } \\
\text { lineari, comprenderne il } \\
\text { funzionamento e saperlo } \\
\text { applicare adottando le } \\
\text { opportune strategie. }\end{array}$ & $\begin{array}{l}\text { Allegati 4, 5, 6 e 7 con problemi } \\
\text { liberamente tratti dal cap. VIII de } \\
\text { "I Nove Capitoli sui procedimenti } \\
\text { matematici" Bacchette e tavole } \\
\text { da calcolo. } \\
\text { Video che mostra il metodo per } \\
\text { sottrazioni ripetute, disponibile } \\
\text { su tablet per ogni gruppo. }\end{array}$ & 6 ore \\
\hline $\begin{array}{l}\text { 4. Verifica e } \\
\text { questionario }\end{array}$ & $\begin{array}{l}\text { Verificare gli apprendimen- } \\
\text { ti e il grado di interesse. }\end{array}$ & $\begin{array}{l}\text { Allegati 8 e 9. } \\
\text { Bacchette e tavole da calcolo. }\end{array}$ & 2 ore \\
\hline
\end{tabular}




\subsection{I numerali nell'antica Cina}

La prima attività si configura come un gioco-sfida per gli studenti: viene chiesto loro di cercare oggetti matematici nella pagina di un antico libro cinese, il "Prezioso Specchio dei Quattro Elementi", contenente il triangolo dei coefficienti binomiali (Figura 9). Nello specifico la richiesta è di argomentare in forma scritta tutte le considerazioni fatte dal gruppo (Allegato 1).

L'obiettivo di questa attività e della successiva, ossia la scoperta del sistema di numerazione cinese e degli algoritmi di calcolo, è un prerequisito per affrontare la procedura del Fangcheng. All'apparenza il compito richiesto si presenta molto alto rispetto alle conoscenze degli studenti, ma la semplicità nascosta nelle relazioni che legano i numeri del triangolo di Tartaglia-Pascal può consentire di superare le difficoltà linguistiche. La richiesta di scoprire un "misterioso" antico testo cinese rende particolarmente stimolante l'attività per una classe di ragazze e ragazzi di 11 anni, certamente più interessante di una lezione frontale introduttiva sul sistema di numerazione cinese.

Figura 9

In questa immagine è riportato il triangolo dei coefficienti fino all'ottava potenza, tratto dal

"Prezioso Specchio dei Quattro Elementi", orientato in accordo con il testo evidenziato nel riquadro in rosso, che si può tradurre con "apri in orizzontale".

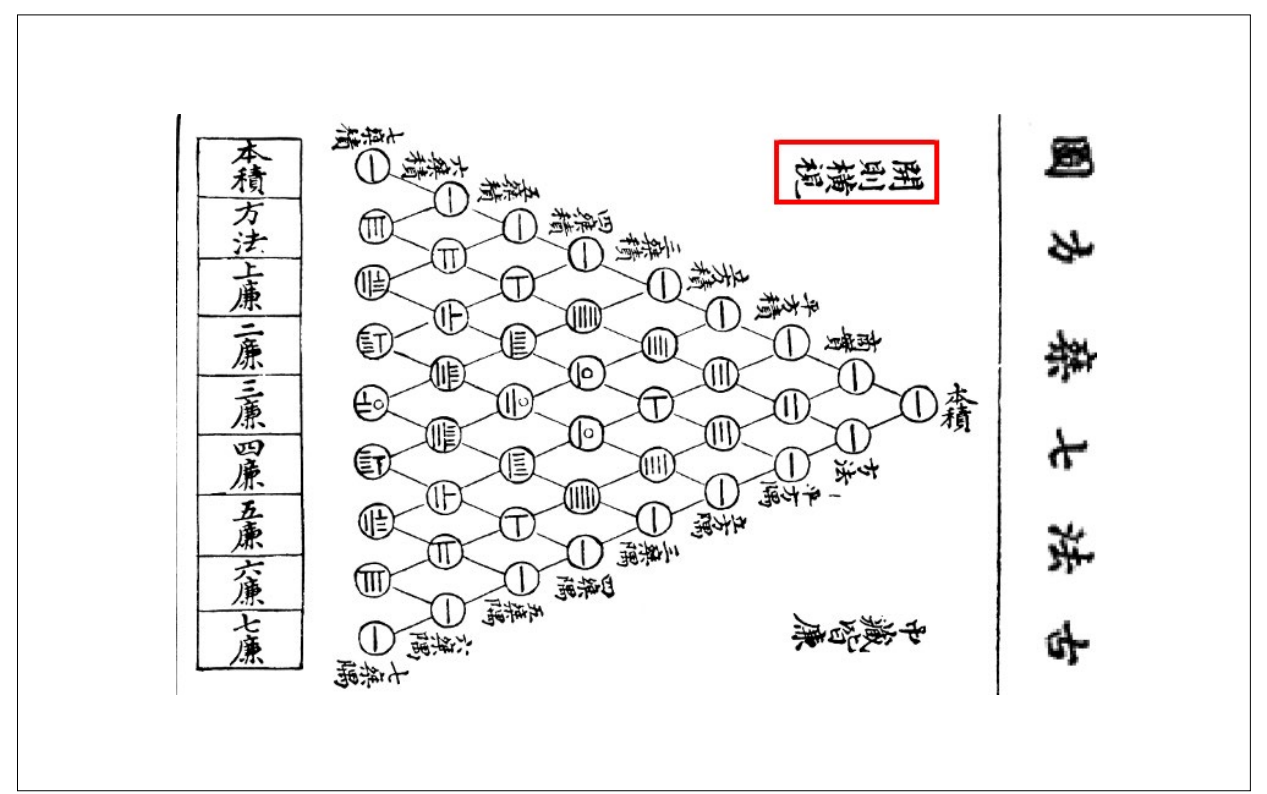

L'immagine originale del triangolo dei coefficienti si presenta con il triangolo orientato con il vertice in alto e la base - che rappresenta i coefficienti per l'ottava potenza - in basso, in modo analogo a come siamo soliti vedere le rappresentazioni del triangolo di Tartaglia-Pascal. Tuttavia, nel "Prezioso Specchio dei Quattro Elementi" il triangolo era orientato con una rotazione di un quarto di giro verso destra, come indica la scritta evidenziata in Figura 9, che si può tradurre con "apri in orizzontale". Di conseguenza, i segni all'interno dei cerchi risultano anch'essi ruotati rispetto alla notazione introdotta nel par. 2.1 e utilizzata nei "Nove capitoli per i procedimenti matematici".

Al fine di ovviare a questo inconveniente e allo stesso tempo mantenere una struttura del triangolo dei coefficienti che gli studenti potranno riconoscere quando studieranno il triangolo di Tartaglia-Pascal, abbiamo scelto di operare una modifica dell'orientamento dei segni all'interno dei cerchi, come si può vedere nell'immagine di Figura 10. 
Figura 10

L'immagine riporta il

triangolo dei coefficienti

fino all'ottava potenza

modificato per l'attività

didattica proposta.

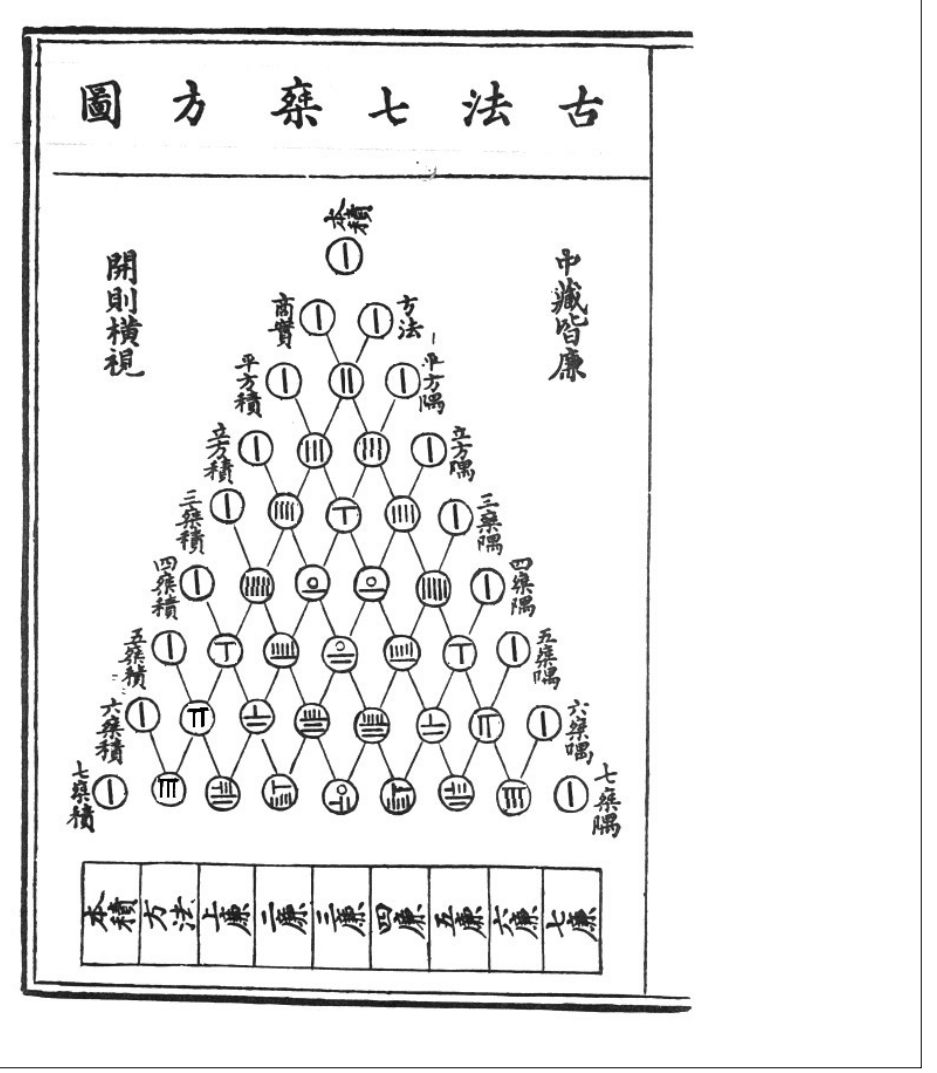

La scelta di sacrificare in parte l'autenticità dell'antico documento è quindi dettata dal mantenere la coerenza sia con lo schema del triangolo di Tartaglia-Pascal, nel quale i numeri presenti in una riga sono dati dalla somma dei numeri adiacenti nella riga soprastante, sia con il sistema di numerazione cinese basato sugli orientamenti Tsung e Heng (cfr. par. 2.1). Si è scelto inoltre di cancellare i segmenti che uniscono i cerchi posti ai lati del triangolo e contenenti il simbolo 1, al fine di rendere più evidente la costruzione dei numerali come somma dei due simboli della riga soprastante.

Dopo l'attività d'indagine sul triangolo è seguita una fase di discussione matematica nella quale i gruppi hanno spiegato cosa hanno scoperto, e l'insegnante ha utilizzato i loro risultati per costruire una tavola di equivalenza tra la notazione cinese antica e quella dei giorni nostri.

Per completezza - e per soddisfare la curiosità degli studenti - al termine della sessione l'insegnante ha illustrato alla classe la traduzione delle parole scritte in cinese intorno al triangolo. Quest'ultima è stata realizzata dagli studenti di nazionalità cinese che frequentano la classe III B della scuola secondaria di primo grado "E. Morelli", facente parte dell'l. C. Torino II di Torino.

\subsection{Le operazioni con bacchette e tavola da calcolo}

In questa fase abbiamo proposto l'apprendimento degli algoritmi di addizione e sottrazione, tramite la visione di due video sulla LIM, che mostrano alcune operazioni eseguite con le bacchette e la tavola da calcolo. Tali video sono stati da noi realizzati, senza commenti, fingendo che il protagonista fosse un insegnante cinese non in 
grado di parlare la nostra lingua. ${ }^{7}$ L'obiettivo è di stimolare la curiosità e consentire di far scoprire in autonomia come funzionano gli algoritmi, soffermandosi in particolare sulle procedure di riporto e di prestito. L'espediente del video ha consentito a noi insegnanti di fingere di non conoscere la procedura e di apprenderla insieme agli studenti; un elemento che ci ha permesso di non uscire dalla modalità laboratoriale. Ad ogni gruppo è stato fornito un set di 50 bacchette di legno (stick da ghiacciolo) e una tavola da calcolo, realizzata disegnando una griglia $4 \times 3$ su un foglio di carta bianco di formato $\mathrm{A} 2$, affinché potessero provare sia a replicare le operazioni del video, sia ad eseguirne di diverse.

Le operazioni mostrate nei video consentono di introdurre gradualmente elementi di difficoltà crescente. Dapprima sono presentate addizioni senza riporto $(122+232$; $217+132$ ), successivamente addizioni con aggiustamenti nello stesso ordine di grandezza $(234+52)$ e infine addizioni con riporto all'ordine di grandezza successivo $(437+154 ; 179+248 ; 647+353)$. Una volta compresa la procedura ed esercitatisi sull'addizione con le bacchette, gli studenti sono passati alle sottrazioni, anch'esse presentate in modo graduale $(197-31 ; 245-126)$.

Compreso il funzionamento della procedura con gli artefatti, abbiamo chiesto agli studenti di argomentare in forma scritta le proprie congetture sull'algoritmo (Allegati 2 e 3). A partire da tali argomentazioni è stata condotta una discussione collettiva, da cui è stato possibile verificare che tutti gli studenti avessero compreso il corretto funzionamento degli algoritmi. In particolare, abbiamo rivolto maggiori attenzioni agli esempi di operazioni che presentavano riporti o prestiti.

\subsection{La procedura del Fangcheng}

Dopo le prime due fasi che introducono gli studenti al sistema di numerazione e di calcolo dell'antica Cina, siamo giunti al cuore della nostra sperimentazione. Anche qui abbiamo seguito l'approccio laboratoriale, partendo da un problema concreto. Abbiamo fornito agli studenti il testo del problema sulle casse e i sacchi di grano (par. 2.2) e abbiamo chiesto loro di provare a risolverlo liberamente, scegliendo il metodo a loro avviso più adeguato (Allegato 4). L'obiettivo è di consentire agli studenti di prendere confidenza con il problema e non tanto - in questa fase - di trovare un metodo di risoluzione generale.

L'Allegato 5 introduce il Fangcheng come un possibile metodo di risoluzione del problema, utilizzato da un contadino di nome Cheng Fang, che aveva letto "I Nove Capitoli sui procedimenti matematici". Gli studenti sono invitati a scoprirne il funzionamento attraverso un video, da noi registrato a velocità accelerata, che mostra come si applica la procedura del Fangcheng. ${ }^{8} \mathrm{Al}$ posto di fornire le due regole su cui si fonda il metodo in maniera procedurale, abbiamo scelto di utilizzare la metafora concettuale delle due bilance a piatti. Infatti, come nella bilancia l'intento è di far sparire da uno dei due piatti un tipo di merce, togliendo quantità equivalenti per rispettare le leggi di equilibrio della bilancia, così nella procedura del Fangcheng la strategia è quella di sottrarre numeri (fisicamente, attraverso le bacchette) al fine di eliminare un'incognita, rispettando i principi di equivalenza delle equazioni.

Gli studenti hanno avuto a disposizione un tablet per ogni gruppo, con il quale han-

7. Video con esempi di addizione: bit.ly/addizioni. Video con esempi di sottrazione: bit.ly/sottrazioni. 8. Video sul Fangcheng: bit.ly/fangcheng. 
Figura 11

In quest'immagine sono associate le due rappresentazioni del sistema

di equazioni: l'equilibrio

fisico della bilancia e

I'uguaglianza con

la tavola da calcolo. no potuto visionare il video utilizzando gli strumenti di navigazione (interrompere, spostarsi avanti e indietro, rallentare...). La richiesta fatta agli studenti è di completare opportunamente la tabella che riportiamo in Figura 11, che mette in relazione il concetto di equilibrio della bilancia con la tavola da calcolo da noi proposta per applicare il Fangcheng.

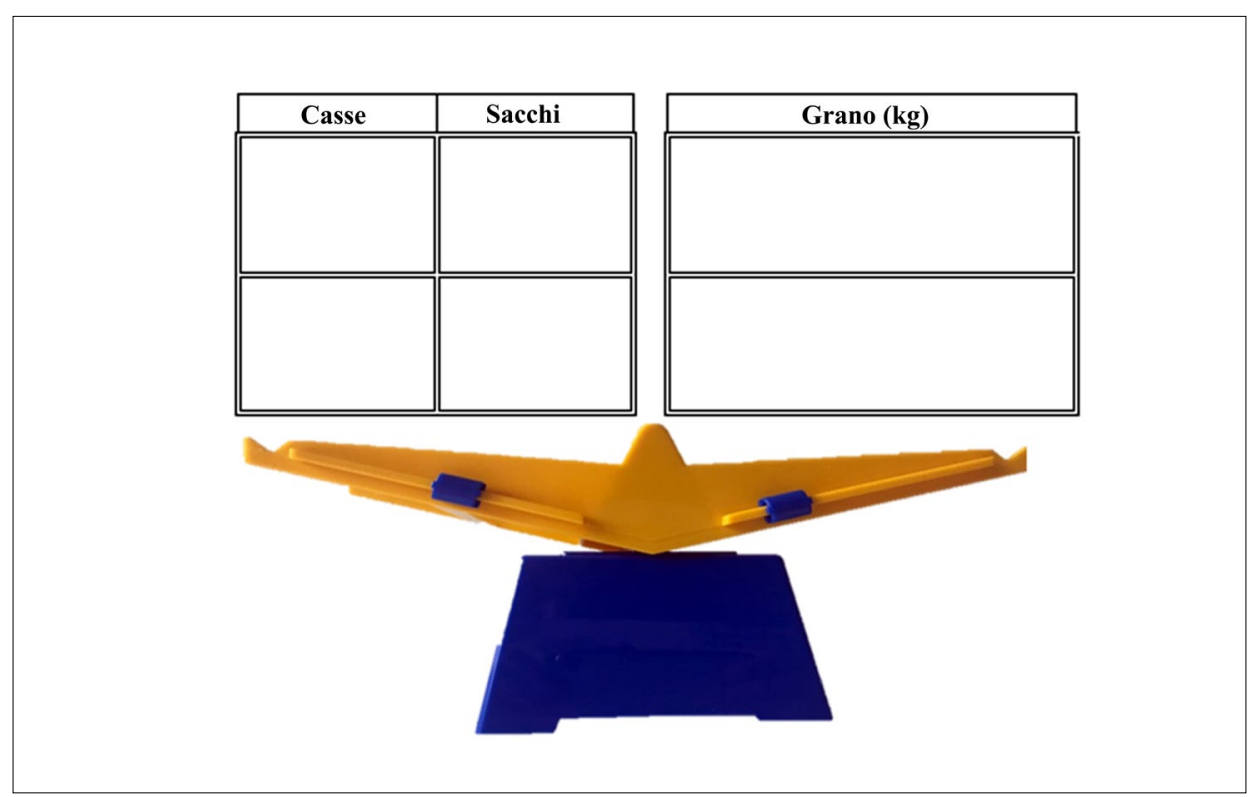

Nell'Allegato 6 abbiamo chiesto agli studenti di provare a ripetere le operazioni eseguite nel video, senza far uso delle bilance, ma utilizzando la notazione cinese con le bacchette e la tavola da calcolo. L'obiettivo è di abbandonare progressivamente I'utilizzo delle bilance in equilibrio per passare alle equazioni rappresentate con i numerali cinesi.

Quando la procedura base è stata compresa nella sua applicazione sull'esempio dato, abbiamo fornito agli studenti una batteria di problemi (Allegato 7), liberamente tratti dall'VIII capitolo del trattato "I Nove Capitoli sui procedimenti matematici". Nello specifico, abbiamo scelto di lasciare invariati i nomi degli oggetti utilizzati nei problemi, e di modificare opportunamente i numeri, in modo che si potesse applicare la "nuova procedura del Fangcheng" per sottrazioni ripetute.

Ad ogni fase di questa attività sul Fangcheng è seguita la discussione matematica con l'intera classe, orchestrata dall'insegnante.

\subsection{Verifica e questionario}

Al termine della sperimentazione, abbiamo proposto agli studenti una verifica scritta (Allegato 8) e un questionario di gradimento delle attività (Allegato 9), al fine di testare il grado di apprendimento della procedura e la percezione che ogni studente aveva del proprio coinvolgimento nell'apprendimento. Gli studenti hanno lavorato individualmente.

Nella verifica abbiamo proposto un problema diverso da quelli incontrati precedentemente e abbiamo chiesto di risolverlo utilizzando il Fangcheng, indicando nelle griglie fornite i calcoli effettuati con le bacchette. Pur avendo a disposizione una 
Figura 12

In questa immagine si osserva una fase dello

svolgimento della verifica, in cui le studentesse

utilizzano l'artefatto delle bacchette per risolvere il problema. quantità sufficiente di bacchette e tavole da calcolo, non le abbiamo volutamente consegnate agli studenti nella fase iniziale, per non indurre la necessità di utilizzarle. Un piccolo gruppo (5 studenti su 20) ha risolto il problema utilizzando la procedura su carta del Fangcheng; gli altri studenti - probabilmente per sentirsi più sicuri nell'esecuzione della procedura - hanno richiesto agli insegnanti la possibilità di utilizzare fisicamente le bacchette e le tavole da calcolo (Figura 12), giungendo alla soluzione entro i tempi stabiliti (1 ora).

Terminato il tempo assegnato per la verifica, abbiamo chiesto loro di compilare un questionario contenente alcune domande di carattere generale sull'attività proposta.

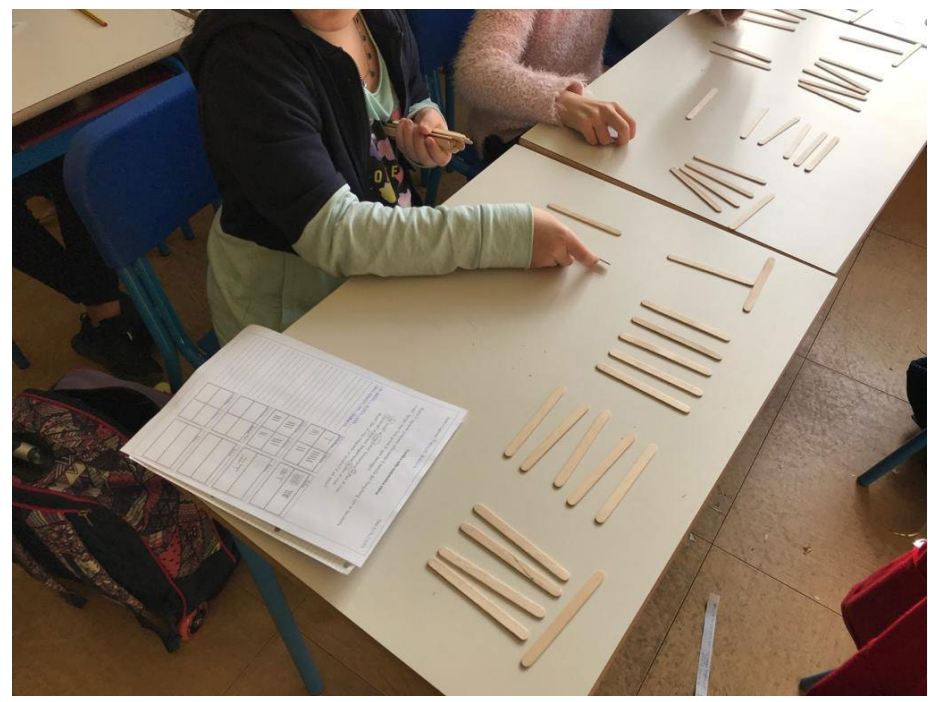

\section{Analisi dei protocolli degli studenti}

Presentiamo in questa sezione l'analisi dei risultati e dei feedback della classe, durante e al termine del percorso didattico.

\subsection{I simboli numerici cinesi e le procedure di calcolo}

Nella prima attività gli studenti sono riusciti a interpretare l'immagine del triangolo dei coefficienti binomiali nonostante le difficoltà linguistiche. Le relazioni tra i simboli numerici sono state riconosciute con ragionamenti differenti. In tutti i gruppi è stato subito associato il simbolo ai numeri da 1 a 5 . In seguito con diverse strategie sono stati decodificati i numeri 6, 7 e 8, con considerazioni legate, ad esempio, alle "diagonali" del triangolo (Figura 13a), all'analogia con i numerali romani («per decifrare i numeri abbiamo fatto finta, che fossero simili ai numeri romani»; «però è sbagliato, perché se fossero numeri romani il 4 si dovrebbe scrivere IV $\gg$ ) e all'orientamento che differenzia le unità (in verticale) dalle decine (in orizzontale).

Fondamentale per la completa traduzione del triangolo è stata la scoperta della relazione additiva tra numeri di righe successive («forse questo qui è un sei, perché noi non abbiamo ancora visto il 6 con questi... perché 3 più 3 è $6 »)$, a cui sono giunti tutti i gruppi dopo varie riflessioni. 
Figura $13 a-13 b$ In queste immagini si osservano diverse strategie per scoprire la notazione cinese: a) l'uso

della diagonale, abbinato alla relazione tra simbolo e quantità: b) il confronto tra somme di ogni riga pari alle potenze successive del 2 .
Figura 14

In queste immagini si osserva come gli studenti scoprano la relazione

additiva, mostrandola con gesti deittici e

verificandola anche con

I'utilizzo della calcolatrice.
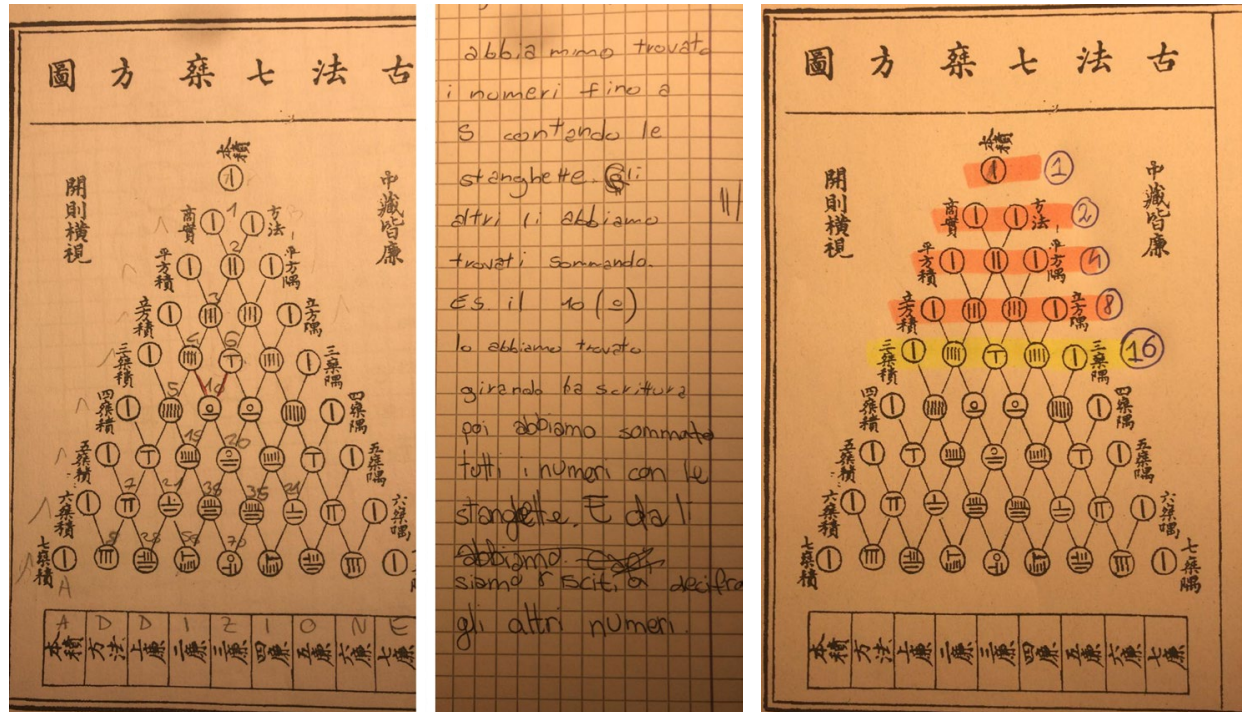

A tal proposito sono interessanti le espressioni e i gesti deittici (Figura 14) che accompagnano le argomentazioni dei ragazzi in questa fase di scoperta (E.: «noi abbiamo calcolato così: questo più questo fa questo. Questa è la nostra logica! E anche qua dice: unisci questi due e fa questo»).
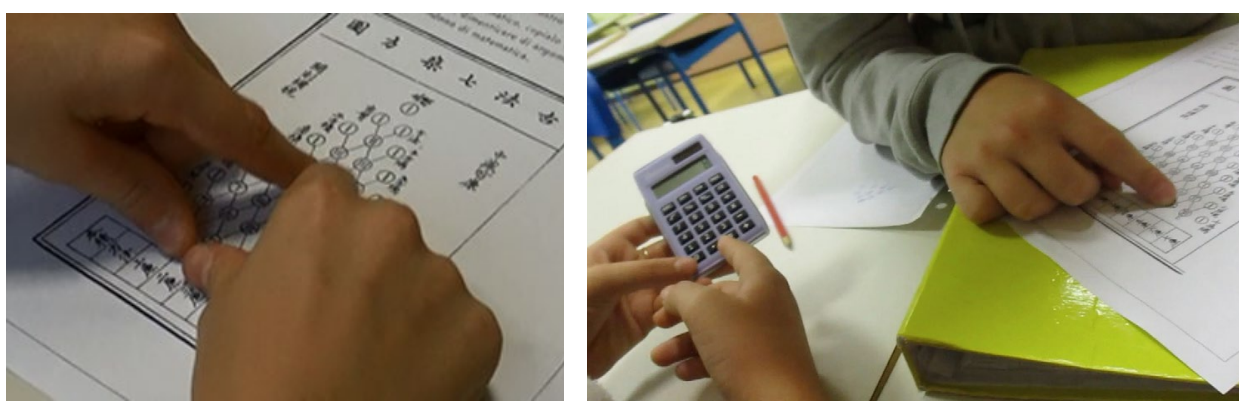

Si noti che, se le strategie precedenti aiutavano a produrre congetture, I'utilizzo della relazione additiva sembra confermarle definitivamente, come dimostrano le argomentazioni finali dei gruppi:

M.: «Fino a 5 ci sono le stanghette, poi da 6 ci sono i numeri strani... [...] Ma tu hai capito?».

D.: «Guarda un attimo, se tu sommi questo, allora il 2, allora 3 più 3 fa 6 , sei più 4 fa 10,10 più 10 fa 20,20 più, questo dovrebbe essere un $15,35 \ldots$.

Alcuni gruppi hanno cercato di ampliare la propria conoscenza dei numerali cinesi, andando oltre quelli riportati nell'immagine, arrivando a comprenderne così il meccanismo di costruzione:

F.: «Questo qua è 7, più 1, 8... non c'è il 9!».

C. «Ferma! Un attimo: e se mettiamo 4 stanghette? Non I'abbiamo ancora fatto. Se metti 4 stanghette... perché nell'8 ce ne sono 3, nel 7 ce ne sono $2 \ldots$ quindi il 9 è una linea con 4 stanghette. Ho scoperto il 9 !». 
Si apprezza il ragionamento induttivo utilizzato dagli studenti, segno di una comprensione della natura operatoria del sistema cinese.

Per la decodificazione del 10 è stata importante la presenza del cerchietto sopra la stanghetta orizzontale, come si evince da alcuni dialoghi:

C.: «Questo qua è un 10, perché uno zero [indicando la stanghetta orizzontale e poi il cerchietto]».

Tutti: «È vero!».

N.: «Quindi se questo qua è un 6 , più 4 fa 10. [...] E questo secondo me è un 20 perché due stanghette e lo zero, sono due decine...».

C.: «più zero...!».

Si tratta di una notazione adottata solamente nella scrittura di alcuni testi, come il "Prezioso Specchio dei Quattro Elementi", e mai con le tavole da calcolo, nelle quali - come anticipato nel par. 2.1 - si lasciava una casella vuota. Abbiamo scelto di mantenere in questa prima fase la rappresentazione dello 0 perché il lavoro è stato condotto su un testo scritto e per velocizzare le fasi preliminari. Successivamente, spostando l'attenzione sulla tavola da calcolo, non è stato necessario utilizzare tale simbolo. Agli studenti è stato chiaro il motivo di questa scelta, che è stata giustificata dai professori sia dal punto di vista storico, sia da quello operativo.

Di particolare interesse sono state le considerazioni di un gruppo che ha decodificato la notazione cinese, lavorando sul confronto tra le somme di ogni riga e scoprendo che si trattava delle potenze di 2 fino 256 ( $\mathrm{L}$.: «Nelle prime quattro righe basta sommare i bastoncini e sono $1,2,4,8$; allora abbiamo pensato che la quinta riga era 16. Allora quel simbolo a forma di T deve essere un 6 ! Perché $1+4+T+4+1$ fa 16 . E così anche le altre righe sotto»). Ciò dipende dal fatto che il triangolo rappresenta $\mathrm{i}$ coefficienti binomiali del quadrato di un binomio $(a+b)^{n}$, che indica le potenze di 2 quando $a=b=1$ (Figura 13b).

Nella seconda attività la visione dei video sull'addizione e sulla sottrazione ha velocizzato la comprensione delle procedure di calcolo, che tuttavia solo all'apparenza sono sembrate semplici agli studenti. Come prevedevamo, sono infatti emerse alcune problematiche nei casi in cui compariva il prestito o il riporto. Durante la sperimentazione ci siamo resi conto che l'iconicità delle bacchette ha aiutato ad affrontare tali ostacoli e a comprenderne meglio il funzionamento odierno a livello concettuale. Troviamo conferma di questo nella seguente significativa frase estratta dal questionario finale: "Con questi numeri cinesi ho fatto più fatica a fare le addizioni, quindi le ho imparate meglio». Riteniamo che tale frase possa essere letta sotto la lente della trasposizione culturale e ci permetta di concludere che l'utilizzo delle bacchette ha effettivamente contribuito a ripensare e aumentare la consapevolezza sugli algoritmi aritmetici della propria cultura, attraverso l'incontro con la cultura cinese antica.

\subsection{La procedura del Fangcheng}

Nell'attività introduttiva al Fangcheng (Allegato 4), in cui occorreva risolvere in autonomia il problema, in tutti i gruppi gli studenti hanno operato in maniera empirica, cercando di trovare la soluzione per tentativi. Nella maggior parte dei casi l'uso di un disegno con l'esatta quantità di casse e sacchi ha aiutato a comprendere il testo e, quindi, a trovare velocemente la soluzione. In alcuni gruppi abbiamo potuto osservare un primo tentativo di confrontare le due equazioni con la sottrazione termine 
Figura 15

In questi protocolli si possono notare due tentativi di risoluzione del problema con l'intuizione del principio base del metodo del Fangcheng per sottrazioni ripetute, realizzati con un linguaggio pre-formale ad astrazione crescente. a termine - regola alla base del metodo per sottrazioni successive del Fangcheng - senza tuttavia riuscire a spiegare le ragioni che li hanno spinti ad effettuare tale passaggio (Figura 15).
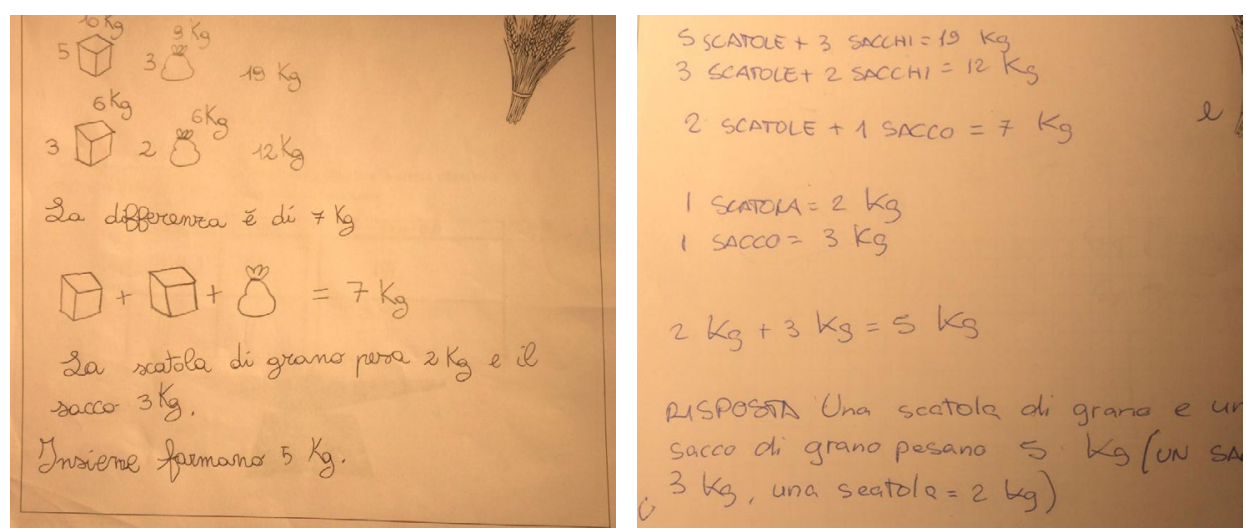

La seconda fase dell'attività, quella in cui è stato mostrato il video in cui si applicava il Fangcheng con le bilance, ha comportato alcune difficoltà iniziali, emerse grazie al confronto con i compagni e con gli insegnanti. Tra le cause non legate al problema matematico in sé possiamo annoverare la velocità del video, per la quale si è adottata la soluzione di rallentare la riproduzione con gli strumenti messi a disposizione dall'app YouTube. Inoltre, i chilogrammi erano rappresentati da monetine ed era impossibile distinguere a colpo d'occhio la numerosità degli oggetti contenuti nelle bilance. ${ }^{9}$ I gruppi che, nella fase precedente, avevano ragionato empiricamente per trovare la soluzione del problema hanno inizialmente faticato a comprendere il nesso tra la quantità di casse, sacchi e chilogrammi tolti da una bilancia e il numero presente nell'altra bilancia. Per questo motivo è stato necessario soffermarsi molto su questa fase, facendo lavorare i ragazzi in modo combinato usando video su tablet, bacchette e tavola da calcolo cartacea (Figura 16). Spendere ulteriore tempo per sciogliere questo nodo si è rivelato utile, infatti tutti i gruppi hanno infine compreso la relazione tra le varie quantità presenti nelle bilance e la soluzione finale del problema.
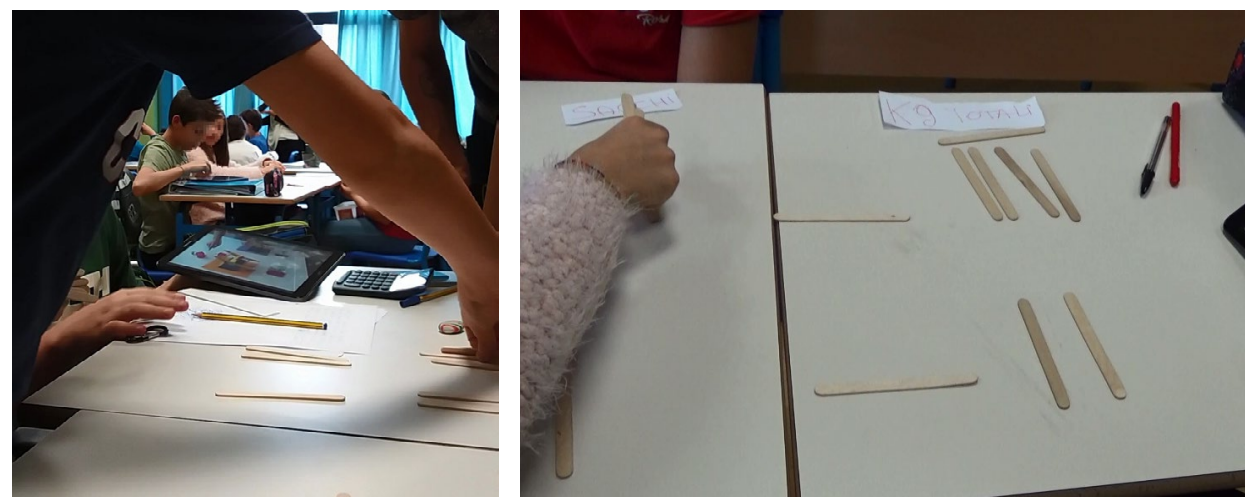

9. Nonostante una ripresa dall'alto avrebbe aiutato a contare velocemente il contenuto di ogni piatto delle bilance, abbiamo preferito realizzare il video in un piano leggermente rialzato rispetto a quello delle bilance, per mostrarne l'equilibrio. 
La discussione matematica ha contribuito a chiarire i dubbi sulla procedura, che alcuni gruppi ancora avevano dopo aver visionato il video. Questa è stata condotta facendo argomentare i passaggi agli studenti, utilizzando la lavagna di ardesia e delle bacchette con adesivi magnetici (Figura 17). In questa fase è stata importante l'enfasi posta dal professore sul legame tra ogni passaggio della procedura e l'equilibrio delle bilance. Queste ultime, infatti, aiutano a controllare la correttezza della procedura, ma essendo una metafora - dalla quale a un certo punto è necessario uscire - non ci si può aspettare una tale verifica ogniqualvolta si operi con la tavola da calcolo. Una delle difficoltà degli studenti è proprio riuscire a mantenere il controllo sull'equilibrio della bilancia anche quando essa non è fisicamente presente. Nella discussione matematica i gruppi generalmente comprendono e sanno argomentare la necessità di ridurre per sottrazioni successive di termini corrispondenti, ma spesso dimenticano di togliere il corrispettivo peso nella colonna dei chilogrammi; I'intervento del professore risulta quindi fondamentale. Proponiamo qui un estratto di discussione (Ins. sta per Insegnante):

M.: «Dobbiamo togliere 3 casse e 2 sacchi da sopra, perché sotto ci sono 3 casse e 2 sacchi».

Ins.: «Ma non basta!».

E.: «Dobbiamo togliere da 19 i 12 chili».

Ins.: «E perché siamo certi che togliendo da qua [indicando 5] questo [3] e da qua [3] questo [2] e da qua [19] questo [12], rimane in equilibrio?».

E.: «Perché sotto ci sono 3 casse e 2 sacchi e togliendo... e la logica del contadino fa che ogni volta bisogna togliere quelli lì [indicando la seconda riga] da quelli sopra e poi quelli sopra a quelli lì».

Ins.: «Ma perché funziona?».

U.: $\quad$ «Perché sai che quelli della seconda bilancia sono uguali. Praticamente sappiamo che le casse e i sacchi pesano 12 chili, quindi se facciamo quello, cioè se facciamo 5 meno 3 e 3 meno 2, sappiamo che è giusto perché anche dall'altra... perché sono lo stesso! Sono 12 chili comunque».

Ins.: «Avete capito? Che cosa stiamo togliendo di qua? Di qua [indicando 19] stiamo togliendo 12 chili. Che forma hanno i 12 chili che togliamo anche di qua [indicando la prima riga] per tenere in equilibrio la bilancia?».

E.: «Casse e sacchi».

Ins.: «E chi mi dice che 3 casse e 2 sacchi pesano 12 chili?».

E.: «Lo dice la bilancia di sotto!».

Figura 17

In questa immagine, tratta dalla discussione collet-

tiva, gli studenti indicano

la seconda equazione, in riferimento alla domanda del professore che chiede di spiegare per quale motivo si tolgono dalla prima equazione 3 casse, 2 sacchi e 12 chili.

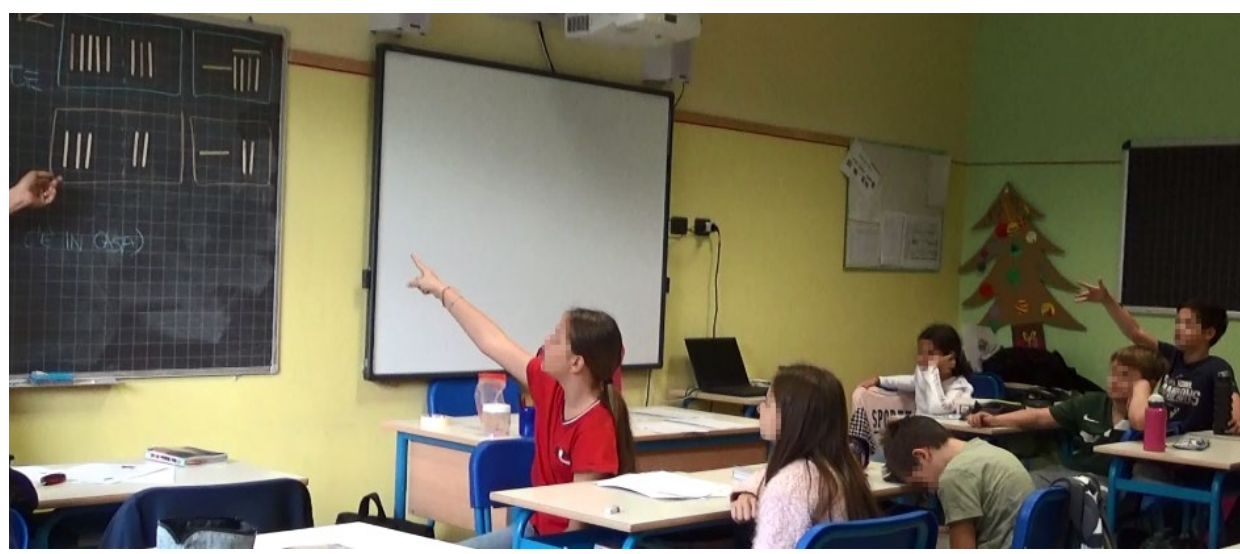


Nella progettazione dell'attività avevamo previsto una fase in cui gli studenti potessero utilizzare fisicamente le bilance, nel caso non avessero compreso la procedura, ma ciò non è stato necessario.

Nell'attività di risoluzione della batteria di problemi (Allegato 7), gli studenti hanno verificato la loro comprensione del metodo. Abbiamo lasciato loro la libertà di utilizzare o meno le bacchette e di adottare la scrittura che preferivano sul foglio (non abbiamo fornito tavole da calcolo cartacee). Diversi gruppi in autonomia hanno lavorato direttamente su quest'ultimo: alcuni hanno disegnato una tavola per ciascun passaggio, altri hanno riportato solo la riga risultante dalla sottrazione. Un gruppo ha invece riportato il disegno dei bastoncini su un'unica griglia e barrando quelli via via eliminati, come se stessero lavorando fisicamente con le bacchette; procedura quest'ultima che necessita di un grande controllo della notazione utilizzata. Si sottolinea a tal proposito uno dei limiti dell'antico algoritmo cinese eseguito con tavole e bacchette, ossia l'impossibilità di memorizzarne i passaggi e quindi di ripercorrerlo all'indietro per verificare i calcoli. Per questo motivo abbiamo scelto di fornire diverse copie della tavola da calcolo, in modo da simulare l'esecuzione con l'artefatto e contemporaneamente avvicinare gli studenti a una prima forma di rappresentazione scritta della risoluzione di un sistema di equazioni.

Si noti che, in alcuni casi, una volta trovata la soluzione con il metodo del Fangcheng, gli studenti verificano il risultato del sistema con le equazioni fornite dal problema; ciò è probabilmente dovuto all'attività empirica precedente e pensiamo possa essere funzionale alla futura comprensione del ruolo dei coefficienti e delle incognite, che essi affronteranno quando incontreranno le equazioni.

Nella discussione matematica finale alcuni studenti hanno risolto uno degli esercizi dell'Allegato 7 in autonomia, giustificando i passaggi con l'uso di considerazioni che fanno riferimento alla bilancia. Si consideri ad esempio il seguente problema $\left(n^{\circ} 5\right.$ nell'Allegato 7):

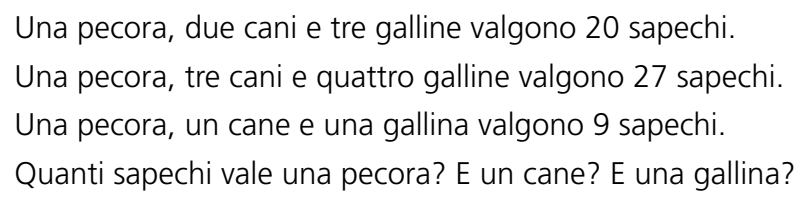

Nel presentare alla lavagna la risoluzione del problema (Figura 18), utilizzando le bacchette magnetiche, U. spiega che per risolvere il problema «bisogna sottrarre dalla bilancia con più peso quella meno pesante. Per esempio, la più grande è quella in centro, poi c'è quella in alto e questa in basso è la meno pesante». Alla richiesta del professore di spiegare da cosa potesse dedurre che quella al centro è più pesante, U. risponde: «sia da qua [indicando le bacchette del piatto a sinistra rappresentanti 1, 3 e 4], sia da qua [indicando 27]». II professore allora ribatte: "Puoi spiegare meglio perché posso togliere il contenuto dei due piatti della bilancia dai due piatti dell'altra?», U.: «perché se stanno su una bilancia che è in equilibrio, vuol dire che hanno lo stesso peso». Quest'ultima affermazione di U. ci suggerisce che lui abbia compreso e interiorizzato il procedimento, riuscendo a mantenere il controllo sia dal punto di vista procedurale, sia da quello concettuale. 
Figura 18

In questa immagine U. sta spiegando la risoluzione del problema 5.

Figura 19

In questa immagine $\mathrm{M}$ sta spiegando la risoluzione del problema 4.
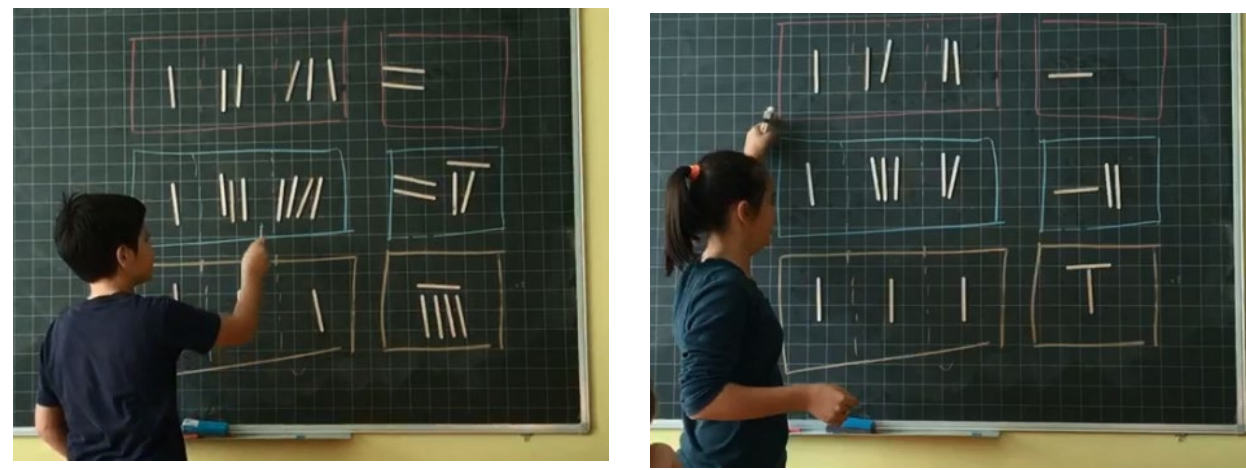

Sottolineiamo, inoltre, che le considerazioni sul peso della bilancia dalla quale partire per eseguire la sottrazione non sono banali, poiché prevedono la comprensione dei termini della sottrazione (minuendo e sottraendo), che spesso gli studenti tendono a invertire; come dimostra il dialogo tra M. e l'Insegnante a proposito della spiegazione di uno dei problemi ( $n^{\circ} 4$ dell'Allegato 7 , Figura 19):
M.: «Dobbiamo partire dalla bilancia blu [seconda equazione] e la sottraiamo a quella rossa».
Ins.: «Quindi da quella rossa sottraiamo la blu?».
M.: «No, sottraiamo quella rossa da quella blu».
Ins.: «E perché possiamo togliere dalla blu e non dalla rossa?».
M.: «Perché la blu contiene più cose, cioè è pesante!».
Ins.: «Ok. Quindi dalla bilancia più pesante cosa togliamo?».
M.: «Tolgo due soldati, due guerrieri e un ufficiale da questo piatto della bilancia e 10 dall'altro piatto, quindi resta $2 »$.
Ins.: «E perché?».
M.: «Perché due soldati, due guerrieri e un ufficiale sono 10 [indicando $\mathrm{i}$ termini della prima equazione]».

Riteniamo che, nonostante non sia sempre esplicitata nella spiegazione dei passaggi, la metafora della bilancia sia risultata efficace nella comprensione dei principi alla base del Fangcheng. L'intervento dell'insegnante per aiutare gli studenti a spostarsi dal piano algebrico dell'uguaglianza a quello fisico della bilancia in equilibrio è servito per verificare che l'automatismo operativo fosse a livello concettuale e non procedurale.

Notiamo, infine, che nella risoluzione di alcuni problemi dell'Allegato 7 e del problema della Verifica, I'applicazione della procedura del Fangcheng conduceva ad un sistema nel quale un'equazione aveva un coefficiente non unitario. Per la risoluzione di tale equazione era dunque richiesto di operare una divisione tra il termine noto e il coefficiente dell'incognita. Tutti gli studenti sono stati in grado di applicare questa procedura, che non era stata presentata nelle attività didattiche, riflettendo sul senso del problema, come possiamo osservare dal protocollo estratto da una verifica, che riportiamo in Figura 20. 
Figura 20

In questo protocollo, che riporta i passaggi finali della verifica, si osserva la giustificazione della

procedura di divisione da attuare al termine della procedura del Fangcheng.

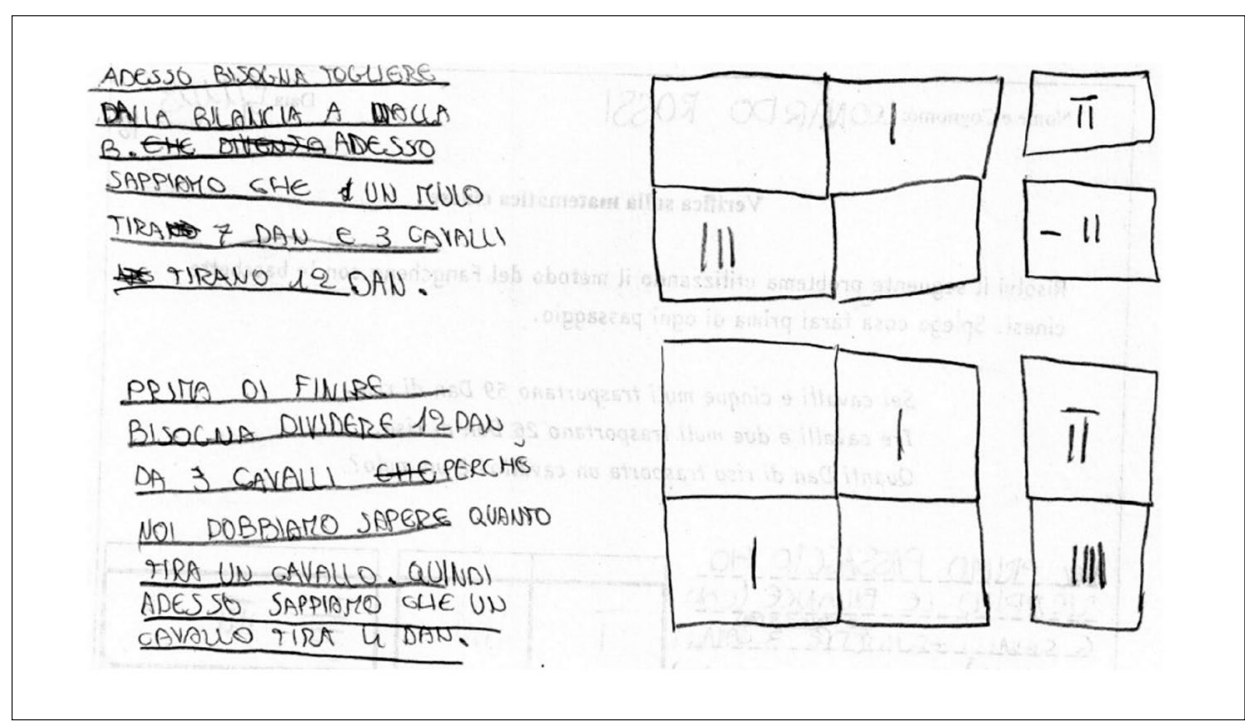

\section{Conclusioni}

Riteniamo che la nostra sperimentazione abbia condotto a buoni risultati nella classe. In termini di apprendimento si evidenzia che 18 studenti su 20 hanno saputo applicare autonomamente la procedura del Fangcheng, risolvendo correttamente il problema proposto nella verifica, di questi - come anticipato nel par. 5.4 - 5 non hanno avuto bisogno di utilizzare l'artefatto delle bacchette e della tavola da calcolo. I due studenti che non hanno portato a termine il compito assegnato in autonomia sono riusciti a risolvere il problema con l'aiuto dell'insegnante. Per quanto concerne, invece, l'atteggiamento nei confronti della matematica, ci possiamo basare sui questionari di gradimento che mostrano un buon coinvolgimento da parte degli studenti; come si evince dalle parole più ricorrenti con cui gli studenti hanno descritto le attività: divertirsi, capire, collaborare, interessante, scoprire.

Gli studenti hanno apprezzato particolarmente I'analisi e la scoperta di un testo storico e di un'antica procedura che i cinesi realmente utilizzavano. Questo ci fornisce un riscontro su quanto la trasposizione culturale sia stata utile per il raggiungimento dei nostri obiettivi: da un lato stimolare l'interesse degli studenti, dall'altro aiutarli a comprendere più in profondità alcuni argomenti fondamentali della matematica, quali le operazioni aritmetiche e i problemi risolvibili con sistemi di equazioni lineari. Nello specifico, relativamente alle operazioni con le bacchette, rileviamo che in generale negli studenti persiste la difficoltà legata al prestito e al riporto nella sottrazione e addizione. Essi sembrano, su tali procedure, preferire riferirsi agli algoritmi appresi con il sistema di numerazione indo-arabico. Tuttavia, dove non c'è il problema del prestito o del riporto, gli studenti scelgono di utilizzare le cifre cinesi, che, grazie alla loro natura operatoria, sono di gran lunga più efficienti. II nostro intento era proprio questo, e non già di sostituire il loro usuale sistema di numerazione, ma di aiutarli a comprenderne meglio il funzionamento. Riteniamo che tra i fattori che hanno inciso sul raggiungimento di questo obiettivo vi sia la componente iconica e indicale dell'artefatto. Per quanto riguarda il metodo del Fangcheng concordiamo con i risultati di Bagni, che nella sua sperimentazione ha riscontrato come gli allievi non apprendano 
mnemonicamente la "regola", ma agiscano strategicamente facendosi aiutare dalla presenza fisica delle bacchette. Ciò risulta ancor più evidente nella nostra sperimentazione che prevedeva esclusivamente il metodo per sottrazioni ripetute e l'uso delle bilance: la procedura di risoluzione seguita dagli allievi è stata validata per ogni passaggio da ragionamenti che facevano riferimento alle leggi fisiche delle bilance. Tale metafora concettuale aiuta a dare un senso alla procedura fondante del metodo, garantendo l'apprendimento di una strategia di risoluzione a livello concettuale e non solo procedurale. Gli artefatti (video su tablet e bilance) hanno contribuito a far comprendere e dare un senso alla procedura del Fangcheng, che è stata oggettualizzata e traslata sulla tavola da calcolo. Quest'ultima è a sua volta I'artefatto, inteso come strumento di mediazione semiotica, che ha aiutato gli studenti ad avvicinarsi ad una prima formalizzazione pre-algebrica dei sistemi di equazioni.

Come anticipato, la procedura delle sottrazioni ripetute è applicabile solamente in alcune tipologie di problemi; mentre la procedura del Fangcheng cosiddetta di «eliminazione per moltiplicazione reciproca» (Di Paola \& Spagnolo, 2009) offre un campo di applicazione più ampio. Per questo motivo potrebbe essere interessante proseguire questa ricerca, applicando la metafora concettuale delle bilance anche all'approccio moltiplicativo. In prospettiva futura sarebbe altrettanto importante come sottolineato dallo stesso Bagni (2009) - approfondire il passaggio dall'esperienza pre-algebrica a quella algebrica con la notazione moderna.

Per il lavoro in classe, questa attività apre interessanti prospettive. Le basi concettuali gettate tramite I'utilizzo degli artefatti potranno essere opportunamente richiamate nel momento dello studio delle equazioni di primo grado e dei principi di equivalenza delle stesse. In tale momento si potrebbe approfondire il ruolo dell'attività qui presentata, degli artefatti utilizzati e dei segni prodotti nel passaggio dall'esperienza pre-algebrica a quella algebrica con la notazione moderna. Inoltre, la comprensione concettuale degli algoritmi di calcolo e dell'uguaglianza nei sistemi di equazioni può essere ulteriormente consolidata tramite attività di peer education tra classi parallele, nelle quali studenti, ormai esperti di storia della matematica cinese, possono insegnare quanto appreso ai compagni. Spingendosi oltre, si può ipotizzare di svolgere un'analoga attività di peer education sia verso i bambini di Scuola Primaria relativamente alle procedure di calcolo, sia verso studenti del primo biennio delle Scuole Secondarie di secondo grado per quanto riguarda i sistemi di equazioni. Questi ultimi, infatti, sarebbero in grado di effettuare un collegamento tra le tecniche di risoluzione dei sistemi apprese in classe e i concetti presentati dagli studenti della scuola secondaria di primo grado.

In definitiva, pensiamo che questa sia una di quelle esperienze significative, di cui si fa menzione nei traguardi per lo sviluppo delle competenze delle Indicazioni Nazionali (MIUR, 2012), che contribuisce a rafforzare «un atteggiamento positivo rispetto alla matematica» e a far capire come "gli strumenti matematici appresi siano utili in molte situazioni per operare nella realtà». Inoltre, aiuta a chiarire come la matematica sia uno strumento di pensiero, costruito, con diverse modalità, attraverso i secoli e nelle varie parti del mondo.

\section{Bibliografia}

Arzarello, F., \& Robutti, O. (2009). Embodiment e multimedialità, nell'apprendimento della matematica, L'insegnamento della matematica e delle scienze integrate, 32 (3), 243- 268. 
Bachtin, M. (2000). L'autore e l'eroe. Teoria letteraria e scienze umane. Torino: Einaudi.

Bagni, G. T. (2006). Bacchette da calcolo e sistemi di equazioni. In L. Giacardi, M. Mosca \& O. Robutti (A cura di), Associazione Subalpina Mathesis. Conferenze e seminari 2005-2006 (pp. 53-62). Torino: Kim Williams Books.

Bagni, G. T. (2009). Interpretazione e didattica della matematica. Una prospettiva ermeneutica. Bologna: Pitagora.

Bartolini Bussi, M. G., \& Mariotti, M. A. (2008). Semiotic mediation in the mathematics classroom: Artifacts and signs after a Vygotskian perspective. In L. English (Ed.), Handbook of International Research in Mathematics Education (second edition). New York, London: Routledge.

Bartolini Bussi, M. G., Mariotti, M. A., \& Ferri, F. (2005). Semiotic Mediation in The Primary School: Dürer's Glass. In H. Hoffmann, J. Lenhard \& F. Seeger (Eds.), Activity and Sign Grounding Mathematics Education (Festschrift for Michael Otte) (pp. 77-90). New York, USA: Springer.

Bartolini Bussi, M.G., Boni, M., \& Ferri, F. (1995). Interazione sociale e conoscenza a scuola: la discussione matematica. Modena: CDE.

Bartolini Bussi, M. G., Sun, X., \& Ramploud, A. (2013). A dialogue between cultures about task design for primary school. In C. Margolinas (Ed.), Task design in mathematics education. Proceedings of ICMI Study 22 (pp. 551-560). Oxford: U.K.

Chemla, K., \& Shuchun, G. (2004). Les neuf chapitres. Le Classique mathématique de la Chine ancienne et ses commentaires. Paris: Dunod.

Costa, C., Alves, J. M., \& Guerra, M. (2015). Ancestral Chinese method for solving linear systems of equations seen by a ten-years-old Portuguese child. In E. Barbin, U. T. Jankvist, \& T. H. Kjeldsen (Eds.), History and epistemology in mathematics education, The Proceedings of the Seventh European Summer University ESU7 (pp. 169-182). Copenhagen: Aarhus University, Danish School of Education.

D'Amore, B., \& Sbaragli, S. (2018). La matematica e la sua storia. II. Dal tramonto greco al medioevo. Bari: Dedalo.

Di Paola, B., \& Spagnolo, F. (2009). I sistemi indeterminati nei "Nove Capitoli" di Liu Hui. II ruolo del "contesto" per determinare I' "algoritmo fondamentale" come strumento argomentativo. Quaderni di ricerca in didattica della matematica, 19, 101-171.

Furinghetti, F., \& Radford, L. (2002). Historical conceptual developments and the teaching of mathematics: from philogenesis and ontogenesis theory to classroom practice. In L. English (Ed.), Handbook of International Research in Mathematics Education (pp. 631-654). Hillsdale: Erlbaum.

Giacomin, A., \& Navarra, G. (2003). Unità 6. Dalla bilancia a piatti all'equazione. Progetto ArAl, 6. Bologna: Pitagora.

Lakoff, G., \& Núñez, R. E. (2000). Where mathematics comes from: How the embodied mind brings mathematics into being. New York: Basic Books.

Malara, N. A., \& Navarra, G. (2003). Quadro teorico di riferimento e glossario. Bologna: Pitagora.

Martzloff, J. C. (2006). A History of Chinese Mathematics. Berlin Heidelberg: Springer-Verlag. 
Mellone, M., \& Ramploud, A. (2015). Additive structure: An educational experience of cultural transposition. In X. Sun, B. Kaur \& N. Novotná (Eds.), Proceedings of the ICMI Study 23 (pp. 567-574). China, Macau: University of Macau.

Mellone, M., Ramploud, A., Di Paola, B., \& Martignone, F. (2019). Cultural transposition: Italian didactic experiences inspired by Chinese and Russian perspectives on whole number arithmetic. ZDM, 51(1), 199-212.

Mihajlović, A., \& Milikić, M. (2019). Fangcheng method as a tool for developing pre-algebra concepts in primary grade student. In S. Lawrence, A. Mihajlović \& O. Đokić (Eds.), Proceedings of the Training Conference History of Mathematics in Mathematics Education, Faculty of Education, University of Kragujevac, Jagodina.

MIUR (2012). Indicazioni nazionali per il curricolo della scuola dell'infanzia e del primo ciclo d'istruzione. Firenze: Le Monnier.

Rabardel, P. (1999). Eléments pour une approche instrumentale en didactique des mathématiques. In M. Bailleul, Actes de la dixième université d'été de didactique des mathématiques (pp. 203-213). Caen: ARDM.

Radford, L. (2003), On Culture and Mind. A post-Vygotskian Semiotic Perspective, with an Example from Greek Mathematical Thought. In M. Anderson, A. Sáenz-Ludlow, S. Zellweger \& V. Cifarelli (Eds.), Educational Perspectives on Mathematics as Semiosis: From Thinking to Interpreting to Knowing (pp. 49-79). Ottawa: Legas.

Radford, L., Boero, P., \& Vasco, C. (2000). Epistemological assumptions framing interpretations of students understanding of mathematics. In J. Fauvel \& J. Van Maanen (Eds.), History in Mathematics Education (pp. 162-167). The ICMI Study. Dordrecht: Kluwer.

Vygotskij, L. S. (1987). I/ processo cognitivo. Torino: Boringhieri.

\author{
Autori/Raffaele Casi ${ }^{\star}$ e Chiara Pizzarelli ${ }^{\circ}$ \\ ^Istituto Comprensivo Andezeno - Andezeno (TO), Italia \\ `Istituto Comprensivo Torino II E. Morelli - Torino, Italia \\ raffaele.casi@istruzione.it, chiara.pizzarelli@unito.it
}

